\title{
Estimating a change point in a sequence of very high-dimensional covariance matrices
}

\author{
H. Dette, G. M. Pan and Q. Yang
}

\begin{abstract}
This paper considers the problem of estimating a change point in the covariance matrix in a sequence of high-dimensional vectors, where the dimension is substantially larger than the sample size.

A two-stage approach is proposed to efficiently estimate the location of the change point. The first step consists of a reduction of the dimension to identify elements of the covariance matrices corresponding to significant changes. In a second step we use the components after dimension reduction to determine the position of the change point. Theoretical properties are developed for both steps and numerical studies are conducted to support the new methodology.
\end{abstract}

Keywords: High-dimensional covariance matrices; change point analysis; dimension reduction.

\section{Introduction}

Change point detection has a long history having it origins in quality control [see Wald (1945) or Page $(1954,1955)$ for early references] and it has been an active field of research until today since the phenomena of sudden changes arise in various areas, such as financial data (house market, stock), signal processing, genetic engineering, seismology, machine learning. In the last decades numerous authors have worked on this problem from several perspectives including the construction of tests for the hypotheses of the existence of change points and the estimation of their locations. We refer to Aue and Horváth (2013) and Jandhyala et al. (2013) for some recent reviews on this subject.

An important problem in the detection of structural breaks in multivariate data is the detection of changes in a sequence of means. Chu et al. (1996), Horváth et al. (1999), 
Horváth and Hušková (2012) and Kirch et al. (2015) investigated this problem using different variants of CUSUM statistics. More recently, the high-dimensional case (dimension larger than the sample size) has been discussed by several authors as well. Jirak (2015) considered a maximum of statistics across panels coordinate-wise to test the hypothesis of at least one change point in a sequence of high dimensional mean vectors [see also Dette and Gösmann (2017) who studied relevant changes in this context]. Cho and Fryzlewicz (2015) suggested sparsified binary segmentation for this problem, while Cho (2016) investigated a double CUSUM approach transferring - roughly speaking - the high-dimensional data to a univariate CUSUM statistic. We also mention the work of Enikeeva and Harchaoui (2014), who looked at the problem under sparse alternatives and Wang and Samworth (2018), who considered the situation, where at certain time points, the mean structure changes in a sparse subset of the coordinates.

While substantial effort has been spent on change point analysis for the multivariate mean, the problem of detecting structural breaks in the covariance matrix has not been studied so intensively in the literature. For a fixed dimension, say $p$, Aue and Horváth (2013) developed nonparametric change point analysis based on the well known CUSUM approach. Dette and Wied (2016) proposed a general approach to detect relevant change points in a parameter of a time series. In an online supplement to this paper a test for a relevant change in the covariance matrix is proposed, where the dimension is also fixed. Recently, Kao et al. (2018) considered the case where the dimension is increasing with the sample size and demonstrated by means of a simulation study that tests of stability of the whole covariance matrix have severe size distortions. As an alternative they proposed and investigated change point analysis based on PCA. In an unpublished preprint Avanesov and Buzun (2016) also looked at the high-dimensional setting and suggested a multiscale approach under sparsity assumptions, while Wang et al. (2017) considered the problem of detecting multiple change points in the situation $p=O(n / \log n)$ (here $n$ is the sample size) and investigated optimality properties of the binary segmentation [see Vostrikova (1981)] and the wild binary segmentation algorithm [see Fryzlewicz (2014)] for localising multiple changes points in a sequence of high dimensional covariance matrices.

The purpose of the present paper is to propose an alternative estimator of the change point in a sequence of very high dimensional covariance matrices and to investigate its theoretical and empirical properties, where we do not impose any sparsity assumptions on the matrices. To be precise, suppose that $\mathbf{x}_{1}, \cdots, \mathbf{x}_{k_{0}}, \mathbf{x}_{k_{0}+1}, \cdots, \mathbf{x}_{n}$ are $p$-dimensional observations with common mean vector $\boldsymbol{\mu}$ and existing covariance matrices. The parameter $k_{0}$ defines the true change point in the structure of the covariance matrices. That is, the first 
$k_{0}$ observations have covariance matrix $\Sigma_{1}=\left(\sigma_{1}(a, b)\right)_{a, b=1, \ldots, p} \in \mathbb{R}^{p \times p}$, while the last $n-k_{0}$ observations have covariance matrices $\Sigma_{2}=\left(\sigma_{2}(a, b)\right)_{a, b=1, \ldots, p} \in \mathbb{R}^{p \times p}$ and $\Sigma_{1} \neq \Sigma_{2}$. We are interested in estimating the point $k_{0}$. One difficulty in dealing with changes in the covariance matrix is the dimensionality since there are $p(p+1) / 2$ positions needed to be compared. This brings in much noisy information when there are many equal components in the matrices $\Sigma_{1}$ and $\Sigma_{2}$, leading to a loss of accuracy in detection of the change point. Taking this consideration into account, we propose to proceed in two steps to identify the change point. First, we apply a dimension reduction technique reducing the dimension from $p(p+1) / 2$ in the original problem to a substantially smaller value, say $m$. Roughly speaking, we only keep the components in the analysis for which a weighted mean of the squared differences of the covariance estimators from the samples $\mathbf{x}_{1}, \cdots, \mathbf{x}_{k}$ and $\mathbf{x}_{k+1}, \cdots, \mathbf{x}_{n}$ exceeds a given threshold (the mean is calculated summing with respect to the different values of the potential change points of $k$ ). Therefore our approach is vaguely related to the estimation of sparse covariance matrices, which has found considerable attention in the literature [see Bickel and Levina (2008), Lam and Fan (2009) or Fan et al. (2016) among many others]. However, in contrast to this work, we do not assume a sparse structure of the covariance matrix, but identify important components by thresholding a weighted sum of the (squared) differences corresponding to all potential samples before and after a postulated change point. In a second step after dimension reduction, we use a CUSUM type statistic based on the reduced components to locate the change point.

An outline of the paper is given as follows. In Section 2, we introduce our main methodology - both the dimension reduction step and the detection step. In particular, a bootstrap method is suggested to select the threshold used for the dimension reduction (see the discussion in Section 2.3). Theoretical results are developed in Section 3, where we prove that (asymptotically) we identify all relevant components correctly and that we estimate the location of the change $k_{0}$ consistently. In Section 4 we investigate the finite sample properties of the new method and demonstrate that it yields precise estimates of the change point in situations, where the dimension is substantially larger than the sample size. We also provide a comparison with two alternative methods which are most similar in spirit to our approach and have recently been proposed in the literature. Finally, all proofs and technical details are deferred to an appendix in Section 5. 


\section{Methodology}

Let $\mathbf{x}_{1}, \cdots, \mathbf{x}_{k_{0}}, \mathbf{x}_{k_{0}+1}, \cdots, \mathbf{x}_{n}$ denote a sample of independent $p$-dimensional random vectors with common mean $\boldsymbol{\mu}$ and existing covariance matrices. The position $k_{0}$ is the "true" change point of the covariance matrices, i.e. the first $k_{0}$ random variables $\mathbf{x}_{1}, \cdots, \mathbf{x}_{k_{0}}$ have covariance matrix $\Sigma_{1}=\left(\sigma_{1}(a, b)\right)_{a, b=1, \ldots, p} \in \mathbb{R}^{p \times p}$, while the last $\left(n-k_{0}\right)$ random variables $\mathbf{x}_{k_{0}+1}, \cdots, \mathbf{x}_{n}$ have covariance matrices $\Sigma_{2}=\left(\sigma_{2}(a, b)\right)_{a, b=1, \ldots, p} \in \mathbb{R}^{p \times p}$ and $\Sigma_{1} \neq \Sigma_{2}$. Our aim is to estimate the location $k_{0}$ of the change. For this purpose we proceed in two steps.

- Step 1 consists of a dimension reduction. If

$$
\hat{\Sigma}_{1}^{k}=\left(\hat{\sigma}_{1}^{k}(a, b)\right)_{a, b=1}^{p}
$$

and $\hat{\Sigma}_{k+1}^{n}=\left(\hat{\sigma}_{k+1}^{n}(a, b)\right)_{a, b=1}^{p}$ denote the respective estimators of the covariance matrices from the data $\mathbf{x}_{1}, \ldots, \mathbf{x}_{k}$ and $\mathbf{x}_{k+1}, \ldots, \mathbf{x}_{n}$, we - roughly speaking - only keep components in the change point analysis for which the quantity

$$
\sum_{k=2}^{n-2} k(n-k)\left(\hat{\sigma}_{1}^{k}(a, b)-\hat{\sigma}_{k+1}^{n}(a, b)\right)^{2}
$$

is sufficiently large.

- Step 2 consists of the detection of a change point in the data obtained after dimension reduction. For this purpose let $\tilde{\sigma}_{1}^{k}$ and $\tilde{\sigma}_{k+1}^{n}$ denote the vectors containing all elements of the matrices $\hat{\Sigma}_{1}^{k}$ and $\hat{\Sigma}_{k+1}^{n}$ corresponding to components which have been identified in the first step of the procedure. Then - roughly speaking - we propose to estimate the change point by maximizing the statistic

$$
\tilde{U}_{n}(k)=\left\|(n-k) k\left(\tilde{\sigma}_{1}^{k}-\tilde{\sigma}_{k+1}^{n}\right)\right\|_{2}^{2}
$$

where $\|\cdot\|_{2}$ denotes the Euclidean norm.

We will give a detailed explanation of these two steps in the following subsections, where the statistics under consideration will be slightly modified. The proposed methodology depends on a regularisation parameter, say $\tau$, determining the amount of dimension reduction for Step 1, and in Section 2.3 we introduce a data-driven method for choosing this threshold.

\subsection{Dimension reduction}

For $i=1, \ldots, n$ denote by $\mathbf{x}_{i}=\left(X_{i 1}, \cdots, X_{i p}\right)^{T}$ the $i$ th observation, let $\overline{\mathbf{x}}=\frac{1}{n} \sum_{i=1}^{n} \mathbf{x}_{i}=$ $\left(\bar{X}_{1}, \cdots, \bar{X}_{p}\right)$ be the sample mean and define

$$
\dot{\mathbf{x}}_{i}=\mathbf{x}_{i}-\overline{\mathbf{x}}=\left(\dot{X}_{i 1}, \ldots, \dot{X}_{i p}\right)^{T}=\left(X_{i 1}-\bar{X}_{1}, \ldots, X_{i p}-\bar{X}_{p}\right)^{T}
$$


as the vector of centered observations. We introduce the following statistic

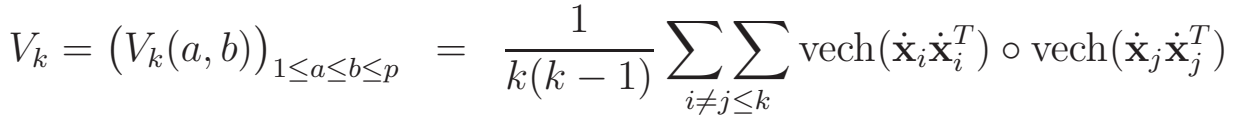

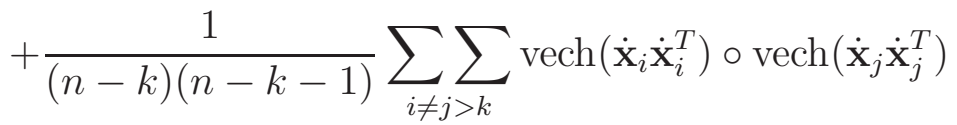

$$
\begin{aligned}
& -\frac{2}{k(n-k)} \sum_{i \leq k} \sum_{j>k} \operatorname{vech}\left(\dot{\mathbf{x}}_{i} \dot{\mathbf{x}}_{i}^{T}\right) \circ \operatorname{vech}\left(\dot{\mathbf{x}}_{j} \dot{\mathbf{x}}_{j}^{T}\right)
\end{aligned}
$$

where "vech $(H)$ " indicates the half-vectorization $p(p+1) / 2$ vector by vectorizing only the lower triangular part of the symmetric matrix $H=(H(a, b))_{a, b=1}^{p}$ and "xoy" is the Hadamard product (or entrywise product) of the vectors $\mathbf{x}$ and $\mathbf{y}$. Obviously, $V_{k}$ is a $p(p+1) / 2$ dimensional vector.

We first give an intuitive illustration of the motivation behind the construction of the statistic $V_{k}$ defined in (2.2), which is in fact motivated by being an approximation of the statistic

$$
\begin{aligned}
\tilde{V}_{k}= & \frac{1}{k^{2}} \sum_{i, j=1}^{k} \operatorname{vech}\left(\dot{\mathbf{x}}_{i} \dot{\mathbf{x}}_{i}^{T}\right) \circ \operatorname{vech}\left(\dot{\mathbf{x}}_{j} \dot{\mathbf{x}}_{j}^{T}\right)+\frac{1}{(n-k)^{2}} \sum_{i, j=k+1}^{n} \operatorname{vech}\left(\dot{\mathbf{x}}_{i} \dot{\mathbf{x}}_{i}^{T}\right) \circ \operatorname{vech}\left(\dot{\mathbf{x}}_{j} \dot{\mathbf{x}}_{j}^{T}\right) \\
& -\frac{2}{k(n-k)} \sum_{i=1}^{k} \sum_{j=k+1}^{n} \operatorname{vech}\left(\dot{\mathbf{x}}_{i} \dot{\mathbf{x}}_{i}^{T}\right) \circ \operatorname{vech}\left(\dot{\mathbf{x}}_{j} \dot{\mathbf{x}}_{j}^{T}\right) \\
= & \left(\frac{1}{k} \sum_{i=1}^{k} \operatorname{vech}\left(\dot{\mathbf{x}}_{i} \dot{\mathbf{x}}_{i}^{T}\right)-\frac{1}{n-k} \sum_{i=k+1}^{n} \operatorname{vech}\left(\dot{\mathbf{x}}_{i} \dot{\mathbf{x}}_{i}^{T}\right)\right) \circ\left(\frac{1}{k} \sum_{i=1}^{k} \operatorname{vech}\left(\dot{\mathbf{x}}_{i} \dot{\mathbf{x}}_{i}^{T}\right)-\frac{1}{n-k} \sum_{i=k+1}^{n} \operatorname{vech}\left(\dot{\mathbf{x}}_{i} \dot{\mathbf{x}}_{i}^{T}\right)\right)
\end{aligned}
$$

Note that the vector $\tilde{V}_{k}$ coincides with the vector

$$
\left(\left(\hat{\sigma}_{1}^{k}(a, b)-\hat{\sigma}_{k+1}^{n}(a, b)\right)^{2}\right)_{1 \leq a \leq b \leq p}
$$

of the squared (componentwise) differences of the elements of the covariance estimators $\hat{\Sigma}_{1}^{k}=\frac{1}{k} \sum_{i=1}^{k} \dot{\mathbf{x}}_{i} \dot{\mathbf{x}}_{i}^{T}$ and $\hat{\Sigma}_{k+1}^{n}=\frac{1}{n-k} \sum_{i=k+1}^{n} \dot{\mathbf{x}}_{i} \dot{\mathbf{x}}_{i}^{T}$. Consequently, at the "true" change point $k=k_{0}$, one can verify that $\tilde{V}_{k_{0}}$ is an estimator of $\operatorname{vech}\left(\Sigma_{1}-\Sigma_{2}\right)^{2}$, which will be used to measure the difference between the two population covariance matrices. The difference between $\tilde{V}_{k}$ and $V_{k}$ consists in the fact that in the statistic $V_{k}$ we omit the terms corresponding to $i=j$ to eliminate the influence of the covariances of the random variables $\operatorname{vech}\left(\dot{\mathbf{x}}_{i} \dot{\mathbf{x}}_{i}^{T}\right)$.

However, in the change point problem we actually do not know the location of $k_{0}$, and we have to consider all the positions $k$ as long as the statistic is well defined. In particular, we obtain for the expectation of the component $V_{k}(a, b)$ of the vector $V_{k}$ corresponding to 
the position $(a, b)$ in the matrices $\Sigma_{1}$ and $\Sigma_{2}$

$$
\mathbb{E} V_{k}(a, b)= \begin{cases}\left(1-\frac{2}{n}\right)^{2}\left(\sigma_{1}(a, b)-\sigma_{2}(a, b)\right)^{2} & \text { if } k=k_{0} \\ \frac{k_{0}\left(k_{0}-1\right)}{k(k-1)}\left(1-\frac{2}{n}\right)^{2}\left(\sigma_{1}(a, b)-\sigma_{2}(a, b)\right)^{2} & \text { if } k>k_{0} \\ \frac{\left(n-k_{0}\right)\left(n-k_{0}-1\right)}{(n-k)(n-k-1)}\left(1-\frac{2}{n}\right)^{2}\left(\sigma_{1}(a, b)-\sigma_{2}(a, b)\right)^{2} & \text { if } k<k_{0}\end{cases}
$$

Note also, that the mean of $V_{k}(a, b)$ always achieves the largest value

$$
\left(1-\frac{2}{n}\right)^{2}\left(\sigma_{1}(a, b)-\sigma_{2}(a, b)\right)^{2}
$$

at the true change point position $k_{0}$ because the coefficients $\frac{k_{0}\left(k_{0}-1\right)}{k(k-1)}$ and $\frac{\left(n-k_{0}\right)\left(n-k_{0}-1\right)}{(n-k)(n-k-1)}$ before $\left(1-\frac{2}{n}\right)^{2}\left(\sigma_{1}(a, b)-\sigma_{2}(a, b)\right)^{2}$ are smaller than 1 when $k \neq k_{0}$. Moreover, these coefficients are only related to $k$ and are not influenced by the position $(a, b)$. Consequently, for any fixed $k$, larger values of $V_{k}(a, b)$ indicate a larger difference between $\sigma_{1}(a, b)$ and $\sigma_{2}(a, b)$, thus implying a significant component. Additionally, instead of investigating each value of $k$ separately, we suggest a weighted sum

$$
D=\frac{1}{n-3} \sum_{k=2}^{n-2} \frac{k(n-k)}{n} V_{k}
$$

to identify the largest components among the $p(p+1) / 2$ entries. The weights $\frac{k(n-k)}{n}$ are introduced to address the different sizes of the variance of $V_{k}$ for different values of $k$. By selecting the largest entries in the vector $D$, we are able to identify the components with the largest changes.

In view of this discussion, we conduct the dimension reduction as follows. Let $D(a, b)$ denote the elements of the vector $D$ corresponding to the position $(a, b)$ in the matrices $\Sigma_{1}$ and $\Sigma_{2}$. We determine all components which are larger than a critical value $\tau$, which will be specified in Section 2.3, define

$$
\mathcal{D}_{\tau}=\{(a, b): D(a, b)>\tau, 1 \leq a \leq b \leq p\}
$$

as the set of all corresponding components and denote by $m=\# \mathcal{D}_{\tau}$ its cardinality. In this way, we reduce the $p(p+1) / 2$-dimensional vector to a vector of dimension $m$. In the next step we will simply work with the $m$-dimensional vectors corresponding to the components identified by the set $\mathcal{D}_{\tau}$. In Theorems 3.1 and 3.2, it will be shown that after dimension reduction with an appropriate threshold all entries with no difference are discarded, while all the entries with a sufficiently large difference are kept. 


\subsection{Change point detection after dimension reduction}

For the estimation of the change point we propose the test statistic

$$
U_{n}(k)=\frac{1}{n^{4}} \sum_{(i \neq t)}^{k} \sum_{=1}^{k} \sum_{(j \neq l)}^{n} \sum_{=k+1}^{n}\left(\dot{\widetilde{\mathbf{x}}}_{i}-\dot{\widetilde{\mathbf{x}}}_{j}\right)^{T}\left(\dot{\widetilde{\mathbf{x}}}_{t}-\dot{\widetilde{\mathbf{x}}}_{l}\right),
$$

where $\dot{\widetilde{\mathbf{x}}}_{i}$ is an $m$-dimensional subvector of $\operatorname{vech}\left(\dot{\mathbf{x}}_{i} \dot{\mathbf{x}}_{i}^{T}\right)$, only keeping the $m$ components of the index set $\mathcal{D}_{\tau}$ defined in (2.4). Then the estimator of the change point $k_{0}$ is defined by

$$
\hat{k}=\underset{1 \leq k \leq n}{\arg \max } U_{n}(k)
$$

The motivation behind the construction of $U_{n}(k)$ stems from the fact the statistic $U_{n}(k)$ is related to a CUSUM type statistic which is frequently used in change point analysis. To be precise, consider the CUSUM statistic

$$
\begin{aligned}
\tilde{U}_{n}(k)= & \frac{1}{n^{4}} \sum_{i, t=1}^{k} \sum_{j, l=k+1}^{n}\left(\dot{\widetilde{\mathbf{x}}}_{i}-\dot{\widetilde{\mathbf{x}}}_{j}\right)^{T}\left(\dot{\widetilde{\mathbf{x}}}_{t}-\dot{\widetilde{\mathbf{x}}}_{l}\right) \\
= & \frac{1}{n^{4}}\left\{(n-k)^{2}\left(\sum_{i=1}^{k} \dot{\widetilde{\mathbf{x}}}_{i}\right)^{T}\left(\sum_{j=1}^{k} \dot{\tilde{\mathbf{x}}}_{j}\right)-2 k(n-k)\left(\sum_{i=1}^{k} \dot{\widetilde{\mathbf{x}}}_{i}\right)^{T}\left(\sum_{j=k+1}^{n} \dot{\widetilde{\mathbf{x}}}_{j}\right)\right. \\
& \left.+k^{2}\left(\sum_{i=k+1}^{n} \dot{\widetilde{\mathbf{x}}}_{i}\right)^{T}\left(\sum_{j=k+1}^{n} \dot{\widetilde{\mathbf{x}}}_{j}\right)\right\} \\
= & \frac{1}{n^{4}}\left\|(n-k) \sum_{i=1}^{k} \dot{\widetilde{\mathbf{x}}}_{i}-k \sum_{j=k+1}^{n} \dot{\widetilde{\mathbf{x}}}_{j}\right\|_{2}^{2}=\frac{1}{n^{4}}\left\|(n-k) k\left(\tilde{\sigma}_{1}^{k}-\tilde{\sigma}_{k+1}^{n}\right)\right\|_{2}^{2}
\end{aligned}
$$

where $\|\cdot\|_{2}$ denotes the usual Euclidean norm and $\tilde{\sigma}_{1}^{k}$ and $\tilde{\sigma}_{k+1}^{n}$ respectively denote the vectors containing the elements of the covariance estimators $\hat{\Sigma}_{1}^{k}$ and $\hat{\Sigma}_{k+1}^{n}$ corresponding to positions identified in the first step. Observing the definition of $\dot{\widetilde{\mathbf{x}}}_{i}$ and noting that the difference

$$
\begin{aligned}
\tilde{U}_{n}(k)-U_{n}(k)= & \frac{1}{n^{4}} \sum_{i=1}^{k} \sum_{j=k+1}^{n}\left\|\dot{\widetilde{\mathbf{x}}}_{i}-\dot{\widetilde{\mathbf{x}}}_{j}\right\|_{2}^{2}+\frac{1}{n^{4}} \sum_{i=1}^{k} \sum_{(j \neq l)=k+1}^{n}\left(\dot{\widetilde{\mathbf{x}}}_{i}-\dot{\widetilde{\mathbf{x}}}_{j}\right)^{T}\left(\dot{\widetilde{\mathbf{x}}}_{i}-\dot{\widetilde{\mathbf{x}}}_{l}\right) \\
& +\frac{1}{n^{4}} \sum_{(i \neq t)=1}^{k} \sum_{j=k+1}^{n}\left(\dot{\widetilde{\mathbf{x}}}_{i}-\dot{\widetilde{\mathbf{x}}}_{j}\right)^{T}\left(\dot{\widetilde{\mathbf{x}}}_{t}-\dot{\widetilde{\mathbf{x}}}_{j}\right)
\end{aligned}
$$

is of smaller order than $U_{n}(k)$ when $k$ is far from 1 and $n$, we see that the statistic $U_{n}(k)$ is a CUSUM type statistic obtained from the components identified in the first step. It is therefore related to the statistic in equation (2.12) in Aue et al. (2009), who proposed an estimator of the change point based on a quadratic form using ALL elements of the 
difference $\hat{\Sigma}_{1}^{k}-\hat{\Sigma}_{k+1}^{n}$. Note that in the definition of $U_{n}$ we eliminate the influence of the covariances $\operatorname{cov}\left(\dot{\widetilde{\mathbf{x}}}_{i}^{T} \dot{\widetilde{\mathbf{x}}}_{i}\right)$ by omitting terms corresponding to $i=t$ and $j=l$ in $\tilde{U}_{n}(k)$. As a consequence, we avoid the estimation of such higher order moments.

We will show in Theorem 3.3 that - under appropriate regularity conditions - the statistic $\hat{k}$ in (2.6) is in fact a consistent estimator of the change point if the dimension and sample size converge to infinity and the threshold is chosen appropriately. More precisely, we can choose $\tau=C \cdot \max (\log p, \log n)$ with a sufficiently large constant $C$ and the dimension $p$ can be of polynomial order of the sample size $n$.

\subsection{Selecting the threshold $\tau$ via resampling}

For a data driven choice of the threshold $\tau$ we propose a bootstrap approach, which mimics the distributional properties in the case of no change point. To be precise define

$$
\begin{aligned}
& \mathbf{T}=\left(\mathbf{T}_{1}, \cdots, \mathbf{T}_{n}\right)=\left(\operatorname{vech}\left(\dot{\mathbf{x}}_{1} \dot{\mathbf{x}}_{1}^{T}\right), \operatorname{vech}\left(\dot{\mathbf{x}}_{2} \dot{\mathbf{x}}_{2}^{T}\right), \cdots, \operatorname{vech}\left(\dot{\mathbf{x}}_{n} \dot{\mathbf{x}}_{n}^{T}\right)\right) \in \mathbb{R}^{\frac{p(p+1)}{2} \times n}, \\
& \mathbf{Z}=\frac{1}{\sqrt{2}}\left(\mathbf{T}_{2}-\mathbf{T}_{1}, \mathbf{T}_{4}-\mathbf{T}_{3}, \ldots, \mathbf{T}_{2\left\lfloor\frac{n}{2}\right\rfloor}-\mathbf{T}_{2\left\lfloor\frac{n}{2}\right\rfloor-1}\right) \in \mathbb{R}^{\frac{p(p+1)}{2} \times\left\lfloor\frac{n}{2}\right\rfloor},
\end{aligned}
$$

and denote by $\mathbf{Z}_{(i)}^{T}$ the $i$-th row of the matrix $\mathbf{Z}$, i.e.

$$
\mathbf{Z}=\left(\mathbf{Z}_{(1)}, \cdots, \mathbf{Z}_{(p(p+1) / 2)}\right)^{T}
$$

For each $\ell=1, \cdots, p(p+1) / 2$, the $\left\lfloor\frac{n}{2}\right\rfloor$-dimensional vector $\mathbf{Z}_{(\ell)}$ can be considered as a combination of $\left\lfloor\frac{n}{2}\right\rfloor$ observations and we denote the empirical standard deviation of these $\left\lfloor\frac{n}{2}\right\rfloor$ observations by $o_{\ell \ell}$. The construction of the matrix $\mathbf{Z}$ ensures that the means of its columns are zero except for at most one position (note that the means of the columns of $\mathbf{T}$ have one change point at $k_{0}$ ), which does not have a substantial effect on the standard deviation provided that the sample size is not too small. If the variance of the random variables in the $\ell$ th row of the matrix $\mathbf{T}$ is constant, it is easy to see that $o_{\ell \ell}^{2}$ estimates this variance. If this assumption is not satisfied, generally speaking, $o_{\ell \ell}^{2}$ always estimates $n \times \operatorname{Var}\left(\bar{T}_{(l)}\right)$, where $\bar{T}_{(l)}$ is the average of the random variables in the $\ell$ th row of the matrix $\mathbf{T}$. Note that the factor $\frac{1}{\sqrt{2}}$ in (2.7) reflects the fact that the matrix $\mathbf{Z}$ is formed from differences of two consecutive columns of the matrix $\mathbf{T}$.

In order to estimate the threshold $\tau$ let $\Upsilon$ denote the $\frac{p(p+1)}{2} \times \frac{p(p+1)}{2}$ diagonal matrix, with entries $o_{11}, \ldots, o_{p(p+1) / 2, p(p+1) / 2}$. We generate a new data matrix $\mathbf{Y}=\left(y_{i j}\right) \in \mathbb{R}^{p(p+1) / 2 \times n}$ with independent standard normal distributed entries and define

$$
\mathbf{X}^{*}=\Upsilon \mathbf{Y}
$$


In other words, in the bootstrap we replace the quantities $\operatorname{vech}\left(\dot{\mathbf{x}}_{1} \dot{\mathbf{x}}_{1}^{T}\right), \ldots, \operatorname{vech}\left(\dot{\mathbf{x}}_{n} \dot{\mathbf{x}}_{n}^{T}\right)$ by the $n$ columns of $\mathbf{X}^{*}$. As a consequence the terms $\dot{X}_{1 a} \dot{X}_{1 b}, \ldots, \dot{X}_{n a} \dot{X}_{n b}$ are replaced by $o_{\ell \ell} y_{\ell 1}, \ldots, o_{\ell \ell} y_{\ell n}$ where the random variables $y_{\ell 1}, \ldots, y_{\ell n}$ are independent standard normal distributed and the index $\ell$ corresponds to the position $(a, b)$.

Next we calculate for the matrix $\mathbf{X}^{*}$ the quantities $V_{k}^{*}$ and $D^{*}$ defined in (2.2) and (2.3) respectively, and obtain the vector

$$
D^{*}=\left(D^{*}(a, b): 1 \leq a \leq b \leq p\right)
$$

The threshold $\tau$ is finally defined as the largest entry of $D^{*}$, i.e.

$$
\tau=\max _{1 \leq a \leq b \leq p} D^{*}(a, b)
$$

\section{$3 \quad$ Asymptotic properties}

In this section we discuss the theoretical properties of our approach. For this purpose we need several assumptions, which will be stated first, beginning with conditions on the distribution of the random vectors $\mathbf{x}_{i}$.

Assumption 3.1. Denote $\mathbf{x}_{i}=\left(X_{i 1}, \cdots, X_{i p}\right), i=1, \cdots, n$. For any $1 \leq a \leq p, X_{i a}$ is a sub-Gaussian random variable, i.e. there are positive constants $C_{1}, C_{2}$ (independent of the indices $i$ and $a$ ) such that for every $t>0$,

$$
\mathbb{P}\left(\left|X_{i a}\right|>t\right) \leq C_{1} e^{-C_{2} t^{2}}
$$

Moreover, the covariance matrices before and after the change point satisfy $\left\|\Sigma_{\nu}\right\|_{o p} \leq M$ $(\nu=1,2)$ for some positive constant $M$, where $\|\cdot\|_{o p}$ denotes the spectral norm.

Our next assumption specifies the size of the change, which can be detected using the threshold $\tau$. Note that the dimension $p$ is increasing with the sample size and a difference between the matrices $\Sigma_{1}$ and $\Sigma_{2}$ might vanish asymptotically if $p, n \rightarrow \infty$ although it is visible for any fixed $p$ (for example if $\Sigma_{1}-\Sigma_{2}=e_{p} e_{p}^{T} / p$ where $e_{p}^{T}=(0, \ldots, 0,1)$ ).

Assumption 3.2. The smallest nonzero entry of the matrix $\Sigma_{1}-\Sigma_{2}$ satisfies

$$
\left|\sigma_{1}(a, b)-\sigma_{2}(a, b)\right|>\lambda \geq C \sqrt{\frac{\tau}{n}} \max \left\{\frac{n^{2}}{\left(n-k_{0}\right)^{2}}, \frac{n^{2}}{k_{0}^{2}}\right\} .
$$

Note that condition (3.1) implies that

$$
\lambda \geq C \sqrt{\frac{\tau}{n}} \max \left\{\frac{n}{n-k_{0}}, \frac{n}{k_{0}}, \sqrt{\frac{n}{k_{0}}}, \sqrt{\frac{n}{n-k_{0}}}, \frac{n}{k_{0}} \sqrt{\frac{n-k_{0}}{k_{0}}}, \frac{n}{n-k_{0}} \sqrt{\frac{k_{0}}{n-k_{0}}}\right\} .
$$


Assumption 3.3. For some small positive constant $c$ we have

$$
\begin{aligned}
p^{2} n & =o\left(e^{c \tau}\right) \\
p^{2} n & =o\left(e^{c n^{\frac{1}{4}} \sqrt{\tau}}\right) \\
p^{2} n^{2} & =o\left(e^{c \sqrt{\tau}}\right) .
\end{aligned}
$$

Theorem 3.1. Recall the definition of the set $\mathcal{D}_{\tau}$ in (2.4) and define

$$
\mathcal{N}=\left\{(a, b): 1 \leq a \leq b \leq p ; \sigma_{1}(a, b)=\sigma_{2}(a, b)\right\}
$$

as the set of indices corresponding to equal elements in the matrices $\Sigma_{1}$ and $\Sigma_{2}$. Then under Assumption 3.1

$$
\mathbb{P}\left\{\bigcup_{(a, b) \in \mathcal{N}}\{D(a, b)>\tau\}\right\}=\mathbb{P}\left(\mathcal{N} \cap \mathcal{D}_{\tau} \neq \emptyset\right) \leq c_{1} p^{2} n\left[e^{-c_{2} \tau}+e^{-c_{2} n^{1 / 4} \sqrt{\tau}}+n e^{-c_{2} \sqrt{\tau}}\right]
$$

where $c_{1}$ and $c_{2}$ are some constants. In particular, if Assumption 3.3 is also satsified

$$
\mathbb{P}\left\{\bigcup_{(a, b) \in \mathcal{N}}\{D(a, b)>\tau\}\right\} \rightarrow 0
$$

i.e, after dimension reduction, all the entries with no difference are discarded.

Theorem 3.2. Recall the definition of the set $\mathcal{D}_{\tau}$ in $(2.4)$ and define

$$
\mathcal{P}=\left\{(a, b): 1 \leq a \leq b \leq p ;\left|\sigma_{1}(a, b)-\sigma_{2}(a, b)\right|>\lambda\right\}
$$

as the set of components which differ by more than $\lambda$. Then under Assumption 3.1 and 3.2 we have

$$
\mathbb{P}\left\{\bigcap_{(a, b) \in \mathcal{P}}\{D(a, b)>\tau\}\right\}=\mathbb{P}\left(\mathcal{P} \subset \mathcal{D}_{\tau}\right) \geq 1-c_{3} p^{2} n\left[e^{-c_{4} \tau}+e^{-c_{4} n^{1 / 4} \sqrt{\tau}}+n e^{-c_{4} \sqrt{\tau}}\right]
$$

where $c_{3}$ and $c_{4}$ are constants. In particular, if Assumption 3.3 is also satisfied

$$
\mathbb{P}\left\{\bigcap_{(a, b) \in \mathcal{P}}\{D(a, b)>\tau\}\right\} \rightarrow 1
$$

i.e, after dimension reduction, all components corresponding to a difference larger than $\lambda$ are kept.

In our next result we establish the asymptotic consistency of the estimator $\hat{k}$. Here and throughout this paper the symbol $\stackrel{i . p \text {. }}{\longrightarrow}$ denotes convergence in probability. 
Theorem 3.3. If Assumptions 3.1 and 3.2 are satisfied, we have

$$
\mathbb{P}\left\{\left|\frac{\hat{k}}{k_{0}}-1\right| \geq \epsilon\right\} \leq c_{5} p^{2} n\left[e^{-c_{6} \tau}+n e^{-c_{6} \sqrt{\tau}}\right]
$$

where $c_{5}$ and $c_{6}$ are constants. In particular, if Assumption 3.3 is also satisfied it follows

$$
\frac{\hat{k}}{k_{0}} \stackrel{i . p .}{\longrightarrow} 1 \text {. }
$$

Corollary 3.1. Theorem 3.1, Theorem 3.2 and Theorem 3.3 are still true if Assumption 3.3 is replaced by the following Assumption 3.4.

Assumption 3.4. Assume that $k_{0}>n^{\epsilon}$ for some $0<\epsilon<1$ and that there exists a positive constant $M<\infty$ such that $p n^{-M} \rightarrow 0$ and $p n e^{-c \tau} \rightarrow 0$ for some sufficiently small positive constant $c$. Note that $M$ could be any large positive constant.

Remark 3.1. Note that we can choose $\tau=C \cdot \max (\log p, \log n)$ in Assumption 3.4, where $C$ is a sufficiently large constant. Then the only requirements are $k_{0}>n^{\epsilon}$ for the location of the change and that the dimension $p$ cannot exceed a polynomial order of the sample size $n$ (but the degree of the polynomial can be arbitrary). Moreover, the inequality (3.1) also qualitatively describes a relation between the location of the change and the size of the differences between the elements of the covariance matrices before and after the change. For example, if $p>n$, we have $\tau=C \cdot \max (\log p, \log n)=C \cdot \log p$ and, if $k_{0}$ is proportional to $n$, this means that the smallest non zero element of the matrix $\Sigma_{1}-\Sigma_{2}$ should satisfy

$$
\left|\sigma_{1}(a, b)-\sigma_{2}(a, b)\right|>C \sqrt{\frac{\log p}{n}}
$$

This is a well-known order to distinguish signal from noise in covariance matrix estimation, see for example Bickel and Levina (2008), who considered covariance estimators based on thresholding.

On the other hand, the choice $\tau=C \cdot \max (\log p, \log n)$ is not possible in Assumption 3.3. However, if we choose $\tau=C \cdot \max \left((\log p)^{2},(\log n)^{2}\right)$ with a sufficiently large constant $C$, there is no restriction on the dimension $p$ and $n$. Now, if $k_{0}$ is proportional to $n$, this means that the smallest non zero element of the matrix $\Sigma_{1}-\Sigma_{2}$ has to satisfy

$$
\left|\sigma_{1}(a, b)-\sigma_{2}(a, b)\right|>C \frac{\max (\log p, \log n)}{\sqrt{n}}
$$

for some constant $C$. This means that the procedure estimates $k_{0}$ consistently even if the differences between the elements of the two matrices are very small. 


\section{Finite sample properties}

In this section we investigate the finite sample properties of the new change point estimator by means of a simulation study and compare our approach with two alternative methods proposed by Aue et al. (2009) and Avanesov and Buzun (2016), which are most similar in spirit as the procedure proposed in the present paper.

To be precise let $r_{0}=k_{0} / n$ be the "true" change point fraction and let $\hat{r}=\hat{k} / n$, where $\hat{k}$ is the new change point estimator defined in (2.6). All the numerical results below are calculated from 200 replications and we obtain the simulated estimates $\hat{r}_{1}, \ldots, \hat{r}_{K}$ of $r_{0}$. In the following discussion we present the mean

$$
\bar{r}=\frac{1}{K} \sum_{i=1}^{K} \hat{r}_{i}
$$

the standard deviation

$$
\operatorname{std}(\hat{r})=\sqrt{\frac{1}{K-1} \sum_{i=1}^{K}\left(\hat{r}_{i}-\bar{r}\right)^{2}}
$$

and the corresponding mean squared error

$$
M S E=\frac{1}{K} \sum_{i=1}^{K}\left(\hat{r}_{i}-r_{0}\right)^{2}=\frac{K-1}{K} \cdot s t d^{2}(\hat{r})+\left(\bar{r}-r_{0}\right)^{2} .
$$

Throughout this section we denote by $\operatorname{blk}(\mathbf{A}, \mathbf{B})$ a block-diagonal matrix composed by matrices $\mathbf{A}$ and $\mathbf{B}$ of appropriate dimension. $\Sigma_{1}=\mathbf{I}_{p}$ is always the identity matrix and we consider four different choices for the matrix $\Sigma_{2}$ to investigate the performance of the new estimator under the following alternatives

- case 1: $\Sigma_{2}=1.5 * \mathbf{I}_{p} ; \quad$ case $2: \Sigma_{2}=2 * \mathbf{I}_{p}$;

- case 3: $\Sigma_{2}=\operatorname{blk}\left(4, \mathbf{I}_{p-1}\right) ; \quad \quad$ case $4: \Sigma_{2}=\operatorname{blk}\left(8, \mathbf{I}_{p-1}\right)$.

Cases 1 and 2 indicate that there are many ( $p$ positions) small disturbances between $\Sigma_{1}$ and $\Sigma_{2}$, with a magnitude increasing from 0.5 to 1 . On the other hand there is only one disturbance between the two population covariance matrices in cases 3 and 4 , but the magnitude is more significant ( 3 and 7 respectively).

The true change point fraction is chosen as $r_{0}=k_{0} / n=0.5$ and the first $k_{0}$ and the last $\left(n-k_{0}\right)$ samples are generated from a multivariate normal distribution $\mathcal{N}_{p}\left(0, \Sigma_{1}\right)$ and a $\mathcal{N}_{p}\left(0, \Sigma_{2}\right)$ distribution, respectively. 


\subsection{Performance of the new estimator}

In order to investigate the finite sample properties of the new estimator we choose two sample sizes $n=100$ and $n=200$ and consider different dimensions $p$ ranging from 5 to 500 . For each pair $(n, p)$, the mean change point fraction, standard deviation (std) and mean squared error (MSE) are recorded for all four cases under consideration, and the results are summarized in Table $1(n=100)$ and Table $2(n=200)$. The numerical results from the two tables can be summarized as follows:

(1) When the sample size $n$ increases, the performance of the estimator is better.

(2) The dimension $p$ of the data does not have a significant influence on the performance of the estimators. In particular the mean squared error is remarkably stable with respect to the dimension in all four cases under consideration.

(3) When the magnitude of the disturbance between $\Sigma_{1}$ and $\Sigma_{2}$ increases, the estimator performs better (compare the results from case 1 with case 2 or from case 3 with case $4)$.

Next we investigate the influence of the dimension reduction step on performance of the estimator. To this end, we consider case 1 and case 3 with sample sizes $n=200$ and present in Table 3 the corresponding results without dimension reduction. In other words we apply the estimator (2.6) based on all components. We note that the computation time without dimension reduction is substantially larger because we work with $p(p+1) / 2$-dimensional vectors.

Comparing Table 3 with the corresponding results in Table 2, we observe the following.

(1) In case 1, the differences in the bias of $\hat{r}$ are negligible (in both cases the mean is very close to 0.5 ). On the other hand the standard deviations and as consequence the MSE in Table 3 are smaller, which means that the estimator without dimension reduction is more stable. Note that there are many small disturbances between two population matrices and thus keeping all positions promotes a safer estimation. On the other hand the MSE results in Table 2 from the estimator using dimension reduction are already satisfactory.

(2) In case 3 when there is only one significant disturbance the situation is different. Although the bias of $\hat{r}$ in Table 3 is smaller, its standard deviation and MSE increase very fast to an unacceptable level with increasing dimension. This means that the estimator without dimension reduction is not reliable if the dimension is large. 
Table 1: Mean, standard deviation (std) and mean squared error (MSE) of the estimator $\hat{r}=\hat{k} / n$ defined in (2.6). The sample size is $n=100$, the change point is $k_{0}=50, \Sigma_{1}=I_{p}$ and results of four different choices for $\Sigma_{2}$ are presented.

\begin{tabular}{c|cccccc||cccccc}
\hline & \multicolumn{9}{|c||}{ case 1 } & \multicolumn{6}{c}{ case 2 } \\
\hline$p$ & 5 & 20 & 60 & 200 & 300 & 500 & 5 & 20 & 60 & 200 & 300 & 500 \\
\hline mean & 0.4985 & 0.5081 & 0.5101 & 0.5213 & 0.5183 & 0.4830 & 0.5186 & 0.5169 & 0.5059 & 0.5017 & 0.5080 & 0.5099 \\
std & 0.1669 & 0.1475 & 0.1274 & 0.1267 & 0.1246 & 0.1766 & 0.1032 & 0.0673 & 0.0780 & 0.0913 & 0.0667 & 0.0797 \\
MSE & 0.0277 & 0.0217 & 0.0162 & 0.0164 & 0.0158 & 0.0313 & 0.0109 & 0.0048 & 0.0061 & 0.0083 & 0.0045 & 0.0064 \\
\hline \hline & \multicolumn{1}{|c|}{ case 3 } & & & & & & case 4 & & \\
\hline$p$ & 5 & 20 & 60 & 200 & 300 & 500 & 5 & 20 & 60 & 200 & 300 & 500 \\
\hline mean & 0.5423 & 0.5416 & 0.5393 & 0.5275 & 0.5378 & 0.5410 & 0.5345 & 0.5288 & 0.5271 & 0.5325 & 0.5317 & 0.5312 \\
std & 0.0570 & 0.0654 & 0.0649 & 0.0491 & 0.0569 & 0.0580 & 0.0471 & 0.0364 & 0.0527 & 0.0389 & 0.0416 & 0.0457 \\
MSE & 0.0050 & 0.0060 & 0.0057 & 0.0031 & 0.0046 & 0.0050 & 0.0034 & 0.0022 & 0.0035 & 0.0026 & 0.0027 & 0.0030 \\
\hline
\end{tabular}

Table 2: Mean, standard deviation (std) and mean squared error (MSE) of the estimator $\hat{r}=\hat{k} / n$ defined in (2.6). The sample size is $n=200$, the change point is $k_{0}=100, \Sigma_{1}=I_{p}$ and results of four different choices for $\Sigma_{2}$ are presented.

\begin{tabular}{|c|c|c|c|c|c|c|c|c|c|c|c|c|}
\hline \multirow[b]{2}{*}{$p$} & \multicolumn{6}{|c|}{ case 1} & \multicolumn{6}{|c|}{ case 2} \\
\hline & 5 & 20 & 60 & 200 & 300 & 500 & 5 & 20 & 60 & 200 & 300 & 500 \\
\hline mean & 0.5108 & 0.5064 & 0.5071 & 0.5058 & 0.5067 & 0.5042 & 0.5105 & 0.5052 & 0.5029 & 0.5010 & 0.5019 & 0.5010 \\
\hline std & 0.1096 & 0.0625 & 0.0437 & 0.0575 & 0.0457 & 0.0529 & 0.0249 & 0.0128 & 0.0120 & 0.0032 & 0.0103 & 0.0043 \\
\hline \multirow[t]{2}{*}{ MSE } & 0.0121 & 0.0039 & 0.0020 & 0.0033 & 0.0021 & 0.0028 & 0.0007 & 0.0002 & 0.0002 & 0.0000 & 0.0001 & 0.0000 \\
\hline & \multicolumn{6}{|c|}{ case 3} & \multicolumn{6}{|c|}{ case 4} \\
\hline$p$ & 5 & 20 & 60 & 200 & 300 & 500 & 5 & 20 & 60 & 200 & 300 & 500 \\
\hline mean & 0.5227 & 0.5234 & 0.5253 & 0.5238 & 0.5211 & 0.5227 & 0.5192 & 0.5175 & 0.5156 & 0.5203 & 0.5212 & 0.5180 \\
\hline std & 0.0320 & 0.0381 & 0.0418 & 0.0350 & 0.0378 & 0.0364 & 0.0307 & 0.0242 & 0.0235 & 0.0255 & 0.0288 & 0.0262 \\
\hline MSE & 0.0015 & 0.0020 & 0.0024 & 0.0018 & 0.0019 & 0.0018 & 0.0013 & 0.0009 & 0.0008 & 0.0011 & 0.0013 & 0.0010 \\
\hline
\end{tabular}

(3) As an interesting phenomenon we note that in contrast to Table 2 the standard deviation and mean squared error in Table 3 show a downward trend in case 1 but an upward tendency in case 3. This observation can be explained by the fact that without dimension reduction the gap between $\Sigma_{1}$ and $\Sigma_{2}$ increases with the dimension $p$ in case 1 but decreases in case 3 .

\subsection{Comparison with an estimator based on a quadratic form}

We first compare the new method with the estimator (2.12) suggested in Aue et al. (2009). Note that this statistic involves an inverse matrix $\hat{\Sigma}_{n}^{-1}$, where $\hat{\Sigma}_{n}$ is an estimator of the long- 
Table 3: Mean, standard deviation (std) and mean squared error (MSE) of the estimator $\hat{r}=\hat{k} / n$ without dimension reduction (i.e. $\hat{k}$ is defined in (2.6) with $\mathcal{D}_{\tau}=\{(a, b): 1 \leq a \leq b \leq p\}$ ). The sample size is $n=200, \Sigma_{1}=I_{p}$ and results for two different choices for $\Sigma_{2}$ are presented (case 1 and case 3) .

\begin{tabular}{c|cccccc||cccccc}
\hline & \multicolumn{6}{|c||}{ case 1 } & \multicolumn{6}{c}{ case 3 } \\
\hline$p$ & 5 & 20 & 60 & 200 & 300 & 500 & 5 & 20 & 60 & 200 & 300 & 500 \\
\hline mean & 0.5226 & 0.5068 & 0.5028 & 0.5037 & 0.5052 & 0.5014 & 0.5265 & 0.5234 & 0.5211 & 0.5097 & 0.5098 & 0.5024 \\
std & 0.0758 & 0.0262 & 0.0193 & 0.0139 & 0.0162 & 0.0101 & 0.0420 & 0.0361 & 0.0429 & 0.1114 & 0.1243 & 0.1614 \\
MSE & 0.0062 & 0.0007 & 0.0004 & 0.0002 & 0.0003 & 0.0001 & 0.0025 & 0.0018 & 0.0023 & 0.0125 & 0.0155 & 0.0259 \\
\hline
\end{tabular}

run covariance satisfying their condition (2.6). Under our setting, this long-run covariance reduces to the population covariance matrix of a $p(p+1) / 2$-dimensional random vector. As consequence the dimension $p$ has to be substantially smaller than the sample size $n$ to estimate the inverse of the covariance matrix precisely. In order to get a larger range for the dimension $p$, we use the sample size $n=400$ in this subsection and let the dimension $p$ vary from 5 to 50 (for larger values of $p$ the method of Aue et al. (2009) shows some instabilties). The location of the change point is assumed to be $k_{0}=200$. In Table 4 we display the results of the estimator in Aue et al. (2009) and the estimator (2.6) proposed in this paper, where we restrict to the case 2 and case 3 for the sake of brevity (the cases 1 and 4 show a very similar picture). We observe that the new estimator always performs better. While this superiority is only minor for small dimension, it becomes substantial for $p=40,50$, in this case the mean squared error of the estimator in (2.12) suggested by Aue et al. (2009) is very large (compared to the cases $p \leq 20$ ), while the new estimate shows a remarkable stability with respect to different dimensions. The differences are also visualized in Figure 1 for the cases 2 and 3, respectively, where we show the histograms of both estimates obtained from the different simulation runs. The sample size is $n=400$, the dimension is $p=25$ and the change point is located at $k_{0}=200$ (red line).

\subsection{Comparison with a multiscale estimator}

We conclude this section with a brief comparison with the procedure in Avanesov and Buzun (2016) who proposed a multiscale approach for the localization of the change point. For the sake of comparison we use the same design as in Section 5.1 of this paper. We also performed a comparison under scenarios considered in Section 4.2. Here the method proposed by these authors does not yield reliable estimates of the change point and the results are not displayed 
Table 4: Mean, standard deviation (std) and mean squared error (MSE) of the estimator (for the relative location $k_{0} / n$ of the change point) proposed in Aue et al. (2009) (left part) and the estimator $\hat{k} / n$ suggested in this paper (right part). The sample size is $n=400, \Sigma_{1}=I_{p}$ and results for two different choices of the matrix $\Sigma_{2}$ are displayed.

\begin{tabular}{c|ccccc||ccccc}
\hline case 2 & \multicolumn{6}{|c||}{ Aue et al. (2009) } & \multicolumn{5}{c}{ estimate (2.6) } \\
\hline$p$ & 5 & 10 & 20 & 40 & 50 & 5 & 10 & 20 & 40 & 50 \\
\hline mean & 0.5099 & 0.5043 & 0.5078 & 0.5800 & 0.6092 & 0.5048 & 0.5022 & 0.5012 & 0.5005 & 0.5003 \\
std & 0.0164 & 0.0103 & 0.0132 & 0.1092 & 0.1184 & 0.0116 & 0.0060 & 0.0037 & 0.0015 & 0.0013 \\
MSE & 0.0004 & 0.0001 & 0.0002 & 0.0183 & 0.0259 & 0.0002 & 0.0000 & 0.0000 & 0.0000 & 0.0000 \\
\hline \hline case 3 & \multicolumn{4}{|c||}{ Aue et al. (2009) } & & \multicolumn{5}{c||}{ estimate $(2.6)$} \\
\hline$p$ & 5 & 10 & 20 & 40 & 50 & 5 & 10 & 20 & 40 & 50 \\
\hline mean & 0.5167 & 0.5148 & 0.5067 & 0.4988 & 0.4909 & 0.5145 & 0.5112 & 0.5128 & 0.5135 & 0.5125 \\
std & 0.0236 & 0.0351 & 0.0387 & 0.1386 & 0.1432 & 0.0270 & 0.0173 & 0.0200 & 0.0197 & 0.0209 \\
MSE & 0.0008 & 0.0014 & 0.0015 & 0.0191 & 0.0205 & 0.0009 & 0.0004 & 0.0006 & 0.0006 & 0.0006 \\
\hline
\end{tabular}
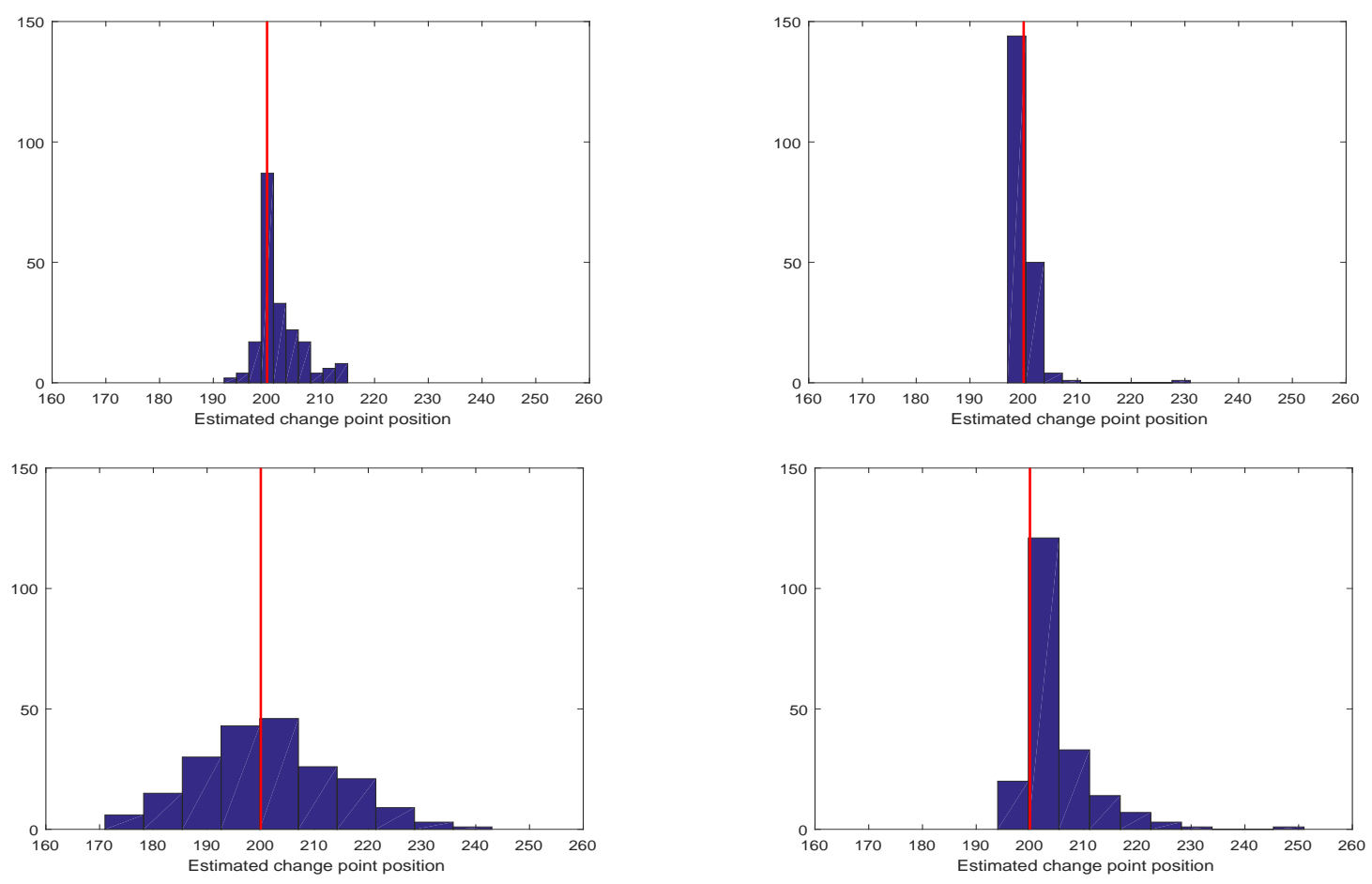

Figure 1: Histograms of estimated change point positions for the estimator of Aue et al. (2009) (left panels) and the new estimator (2.6). Upper row: case 2, lower row: case 3. The sample size is $n=400$, the dimension is $p=25$ and the change point is located at $k_{0}=200$ (red line). 
for the sake of brevity.

Table 5: Comparison of the estimate (2.6) with the change point estimate proposed by Avanesov and Buzun (2016). Both estimates are normalized, that is $\hat{r}=\hat{\tau} / n$, where $\hat{\tau}$ is one of the two estimates. The sample size is $n=1000$, the dimension is $p=50$ and the "true" change point is located at $k_{0} / n=500 / 1000=0.5$.

\begin{tabular}{lcc||ccc}
\hline \multicolumn{2}{c||}{ Avanesov and Buzun (2016) } & \multicolumn{4}{c}{ estimate (2.6) } \\
\hline mean & std & MSE & mean & std & MSE \\
\hline 0.4878 & 0.0948 & 0.0091 & 0.5006 & 0.0087 & 0.0001 \\
\hline
\end{tabular}

To be precise we summarize the setting in Avanesov and Buzun (2016) here briefly. The covariance matrix before the change point is $\Sigma_{1}=\mathbf{I}_{p}$ and the matrix $\Sigma_{2}$ after the change point is generated as follows. First a Poisson distributed random variable $K \sim$ Poiss(3) is generated. Then the matrix $\Sigma_{2}$ is composed as a block-diagonal matrix of $K$ (symmetric) matrices of size $2 \times 2$ with ones on their diagonals and their off-diagonal element drawn uniformly from the set $[-0.6 ;-0.3] \cup[0.3 ; 0.6]$. The remaining $(p-2 k) \times(p-2 k)$ diagonal block of the matrix $\Sigma_{2}$ is the identity matrix and all other elements of $\Sigma_{2}$ are 0 . We consider a sample of $n=1000$ observations with dimension $p=50$, where the "true" change point is given by $k_{0}=n / 2=500$. The procedure of Avanesov and Buzun (2016) also requires the specification of a set $\mathcal{I}_{s}$ corresponding to observations without change points and we use $\mathcal{I}_{s}=[1,2, \cdots, 100]$ (as suggested in their paper) in our simulation. Note also that according to (2.5) in Section 2.3 of Avanesov and Buzun (2016) a change point estimator is only defined if there exists a narrowest window detecting a change-point. This was in 199 of the 200 replications the case.

In Table 5 we show the simulated mean, standard deviation and mean squared error of both estimates for 200 simulation runs. We observe that the estimator proposed in this paper shows a substantially better performance than the multiscale estimator introduced by Avanesov and Buzun (2016). Histograms of the simulated change point positions for both methods in Figure 2 point to the same conclusion.

Acknowledgements. The authors would like to thank M. Stein who typed parts of this manuscript with considerable technical expertise. We are also grateful to V. Avanesov and N. Buzun for making the R-code of their procedure available to us. The work of H. Dette was partially supported by the Deutsche Forschungsgemeinschaft (DFG Research Unit 1735). 

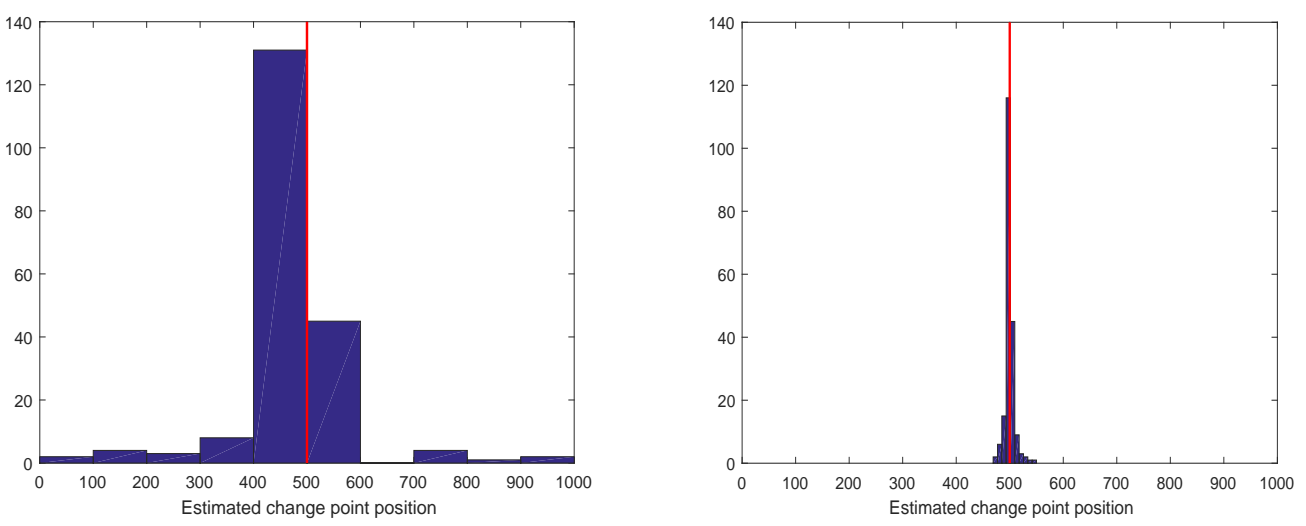

Figure 2: Histograms of the change point estimates proposed by Avanesov and Buzun (2016) (left panel) and the new estimate (2.6) proposed in this paper. The sample size is $n=1000$, the dimension is $p=50$ and the "true" change point is located at $k_{0}=500$ (red line).

Guangming Pan was supported in part by by a MOE Tier 2 grant 2014-T2-2-060 and by a MOE Tier 1 Grant RG25/14 at the Nanyang Technological University, Singapore.

\section{References}

Aue, A., S. Hörmann, L. Horváth, and M. Reimherr (2009). Break detection in the covariance structure of multivariate time series models. Annals of Statistics 37(6B), 4046-4087.

Aue, A. and L. Horváth (2013). Structural breaks in time series. Journal of Time Series Analysis 34(1), 1-16.

Avanesov, V. and N. Buzun (2016). Change-point detection in high-dimensional covariance structure. arXiv:1610.03783v4.

Bickel, P. J. and E. Levina (2008). Covariance regularization by thresholding. Annals of Statistics 36, 2577-2604.

Cho, H. (2016). Change-point detection in panel data via double CUSUM statistic. Electronic Journal of Statistics 10, 2000-2038.

Cho, H. and P. Fryzlewicz (2015). Multiple change-point detection for high-dimensional time series via sparsified binary segmentation. Journal of the Royal Statistical Society, Ser. B 77, 475-507.

Chu, C.-S. J., M. Stinchcombe, and H. White (1996). Monitoring structural change. Econometrica 64(5), 1045-1065.

Dette, H. and J. Gösmann (2017). Relevant change points in high dimensional time series. arXiv:1704.04614v2. 
Dette, H. and D. Wied (2016). Detecting relevant changes in time series models. Journal of the Royal Statistical Society: Series B (Statistical Methodology) 78(2), 371-394.

Enikeeva, F. and Z. Harchaoui (2014). High-dimensional change-point detection with sparse alternatives. arXiv:1312.1900v2.

Fan, J., Y. Liao, and H. Liu (2016). An overview of the estimation of large covariance and precision matrices. Econometrics Journal 19(1), 1-32.

Fryzlewicz, P. (2014). Wild binary segmentation for multiple change-point detection. The Annals of Statistics 42, 2243-2281.

Horváth, L. and M. Hušková (2012). Change-point detection in panel data. Journal of Time Series Analysis 33, 631-648.

Horváth, L., P. Kokoszka, and J. Steinebach (1999). Testing for changes in multivariate dependent observations with an application to temperature changes. Journal of Multivariate Analysis 68(1), 96-119.

Jandhyala, V., S. Fotopoulos, I. MacNeill, and P. Liu (2013). Inference for single and multiple change-points in time series. Journal of Time Series Analysis 34 (4), 423-446.

Jirak, M. (2015). Uniform change point test in high dimension. The Annals of Statistics $43(6), 2451-2483$.

Johnson, W. B., G. Schechtman, and J. Zinn (1985). Best constants in moment inequalities for linear combinations of independent and exchangeable random variables. The Annals of Probability 13(1), 234-253.

Kao, C., L. Trapani, and G. Urga (2018). Testing for instability in covariance structures. Bernoulli 24(1), 740-771.

Kirch, C., B. Muhsal, and H. Ombao (2015). Detection of changes in multivariate time series with application to EEG data. Journal of the American Statistical Association 110(511), 1197-1216.

Lam, C. and J. Fan (2009). Sparsistency and rates of convergence in large covariance matrix estimation. Annals of Statistics 37, 4254-4278.

Page, E. S. (1954). Continuous inspection schemes. Biometrika 41, 100-115.

Page, E. S. (1955). Control charts with warning lines. Biometrika 42(1-2), 243-257.

Vershynin, R. (2017). High Dimensional Probability: An Introduction with Applications in Data Science.

Vostrikova, L. J. (1981). Detecting "disorder" in multidimensional random processes. Soviet Mathematics Doklady 24, 55-59.

Wald, A. (1945). Sequential tests of statistical hypotheses. Annals of Mathematical Statis- 
tics 16, 117-186.

Wang, D., Y. Yu, and A. Rinaldo (2017). Optimal covariance change point detection in high dimension. arXiv:1712.09912v1.

Wang, T. and R. J. Samworth (2018). High dimensional change point estimation via sparse projection. Journal of the Royal Statistical Society: Series B (Statistical Methodology) $80(1), 57-83$. 


\section{Proof of main results}

We first introduce some auxiliary results that are frequently used in the proofs. Lemma 5.1 is a direct conclusion of Lemma 2.7.7 in Vershynin (2017).

Lemma 5.1. For any $1 \leq a, b \leq p, X_{i a} X_{i b}$ is a sub-exponential random variable, i.e. there exist positive constants $C_{1}, C_{2}>0$ (which do not depend on the indices $i$ and a) such that for every $t>0$,

$$
\mathbb{P}\left(\left|X_{i a} X_{i b}\right|>t\right) \leq C_{1} e^{-C_{2} t}
$$

Lemma 5.2 (Corollary 2.8.3 in Vershynin (2017)). Let $W_{1}, \cdots, W_{N}$ be independent, mean zero, sub-exponential random variables. Then, there exist positive constants $C_{1}, C_{2}>0$ such that for every $t>0$,

$$
\mathbb{P}\left\{\left|\frac{1}{N} \sum_{i=1}^{N} W_{i}\right|>t\right\} \leq C_{1} e^{-C_{2} N \cdot \min \left(t^{2}, t\right)} .
$$

Lemma 5.3 (Theorem 2.6.3 in Vershynin (2017)). Let $W_{1}, \cdots, W_{N}$ be independent, mean zero, sub-gaussian random variables. Then, there exist positive constants $C_{1}, C_{2}$ such that for every $t>0$,

$$
\mathbb{P}\left\{\left|\frac{1}{N} \sum_{i=1}^{N} W_{i}\right|>t\right\} \leq C_{1} e^{-C_{2} N t^{2}}
$$

\subsection{Proof of Theorem 3.1}

Observing the construction of the statistic $V_{k}$ in (2.1) and (2.2), we may assume without loss of generality that $\boldsymbol{\mu}=0$. The components of the vector $V_{k}$ and $D$ corresponding to the entry in the position $(a, b)$ of the matrices $\Sigma_{1}, \Sigma_{2}, 1 \leq a, b \leq p$ are given by

$$
\begin{aligned}
V_{k}(a, b)= & \frac{1}{k(k-1)} \sum_{i \neq j \leq k} \sum_{j \leq k}\left(\dot{X}_{i a} \dot{X}_{i b}\right)\left(\dot{X}_{j a} \dot{X}_{j b}\right)+\frac{1}{(n-k)(n-k-1)} \sum_{i \neq j>k} \sum_{i a}\left(\dot{X}_{i a} \dot{X}_{i b}\right)\left(\dot{X}_{j a} \dot{X}_{j b}\right) \\
& -\frac{2}{k(n-k)} \sum_{i \leq k} \sum_{j>k}\left(\dot{X}_{i a} \dot{X}_{i b}\right)\left(\dot{X}_{j a} \dot{X}_{j b}\right) \\
D(a, b)= & \frac{1}{n-3} \sum_{k=2}^{n-2} \frac{k(n-k)}{n} V_{k}(a, b)=D^{(1)}(a, b)+D^{(2)}(a, b)+D^{(3)}(a, b)
\end{aligned}
$$


where $\dot{X}_{i a}=X_{i a}-\bar{X}_{a}=X_{i a}-\frac{1}{n} \sum_{j=1}^{n} X_{j a}$ by (2.1) and the terms $D^{(\ell)}(a, b)$ are defined by

$$
\begin{aligned}
& D^{(1)}(a, b)=\frac{1}{n-3} \sum_{k=2}^{\lfloor\sqrt{n}\rfloor} \frac{k(n-k)}{n} V_{k}(a, b), \\
& D^{(2)}(a, b)=\frac{1}{n-3} \sum_{k=\lfloor\sqrt{n}\rfloor+1}^{n-\lfloor\sqrt{n}\rfloor-1} \frac{k(n-k)}{n} V_{k}(a, b), \\
& D^{(3)}(a, b)=\frac{1}{n-3} \sum_{k=n-\lfloor\sqrt{n}\rfloor}^{n-2} \frac{k(n-k)}{n} V_{k}(a, b) .
\end{aligned}
$$

The reason for this decomposition of $D(a, b)$ is that all the $k$ 's or $(n-k)$ 's in $D^{(2)}(a, b)$ are sufficiently large, while both $D^{(1)}(a, b)$ and $D^{(3)}(a, b)$ only involve $(\lfloor\sqrt{n}\rfloor-1)$ terms and thus the coefficient $\frac{1}{n-3}=\frac{\lfloor\sqrt{n}\rfloor-1}{n-3} \cdot \frac{1}{\lfloor\sqrt{n}\rfloor-1}$ gives us an extra factor of order $\frac{1}{\sqrt{n}}$ in the calculations.

To be precise, let

$$
\begin{array}{ll}
\bar{X}_{k(a, b)}=\frac{1}{k} \sum_{i=1}^{k} X_{i a} X_{i b}, & \bar{Y}_{k(a, b)}=\frac{1}{n-k} \sum_{i=k+1}^{n} X_{i a} X_{i b}, \\
\bar{X}_{k a}=\frac{1}{k} \sum_{i=1}^{k} X_{i a}, & \bar{Y}_{k a}=\frac{1}{n-k} \sum_{i=k+1}^{n} X_{i a}, \\
\dot{\bar{X}}_{k(a, b)}=\frac{1}{k} \sum_{i=1}^{k} \dot{X}_{i a} \dot{X}_{i b}, & \dot{\bar{Y}}_{k(a, b)}=\frac{1}{n-k} \sum_{i=k+1}^{n} \dot{X}_{i a} \dot{X}_{i b}
\end{array}
$$

and

$$
\begin{aligned}
& V_{k 1}=\frac{k(n-k)}{n}\left[\dot{\bar{X}}_{k(a, b)}-\dot{\bar{Y}}_{k(a, b)}\right]^{2}, \quad V_{k 2}=\frac{k(n-k)}{n}\left[\frac{1}{k-1} \dot{\bar{X}}_{k(a, b)}^{2}+\frac{1}{n-k-1} \dot{\bar{Y}}_{k(a, b)}^{2}\right] \\
& V_{k 3}=\frac{k(n-k)}{n}\left[\frac{1}{k(k-1)} \sum_{i=1}^{k}\left(\dot{X}_{i a} \dot{X}_{i b}\right)^{2}+\frac{1}{(n-k)(n-k-1)} \sum_{i=k+1}^{n}\left(\dot{X}_{i a} \dot{X}_{i b}\right)^{2}\right]
\end{aligned}
$$

then a straightforward but tedious calculation yields

$$
\begin{gathered}
\dot{\bar{X}}_{k(a, b)}=\bar{X}_{k(a, b)}-\bar{X}_{k a} \bar{X}_{b}-\bar{X}_{k b} \bar{X}_{a}+\bar{X}_{a} \bar{X}_{b}, \\
\dot{\bar{Y}}_{k(a, b)}=\bar{Y}_{k(a, b)}-\bar{Y}_{k a} \bar{X}_{b}-\bar{Y}_{k b} \bar{X}_{a}+\bar{X}_{a} \bar{X}_{b}
\end{gathered}
$$

and

$$
\frac{k(n-k)}{n} V_{k}(a, b)=V_{k 1}+V_{k 2}-V_{k 3} .
$$

With these notations we decompose the quantities $D^{(i)}(a, b)$ as follows:

$$
D^{(i)}(a, b) \triangleq A^{(i)}+B^{(i)}-C^{(i)}
$$


where

$$
\begin{aligned}
& A^{(1)}=\frac{1}{n-3} \sum_{k=2}^{\lfloor\sqrt{n}\rfloor} V_{k 1}, \quad B^{(1)}=\frac{1}{n-3} \sum_{k=2}^{\lfloor\sqrt{n}\rfloor} V_{k 2}, \quad C^{(1)}=\frac{1}{n-3} \sum_{k=2}^{\lfloor\sqrt{n}\rfloor} V_{k 3}, \\
& A^{(2)}=\frac{1}{n-3} \sum_{k=\lfloor\sqrt{n}\rfloor+1}^{n-\lfloor\sqrt{n}\rfloor-1} V_{k 1}, \quad B^{(2)}=\frac{1}{n-3} \sum_{k=\lfloor\sqrt{n}\rfloor+1}^{n-\lfloor\sqrt{n}\rfloor-1} V_{k 2}, \quad C^{(2)}=\frac{1}{n-3} \sum_{k=\lfloor\sqrt{n}\rfloor+1}^{n-\lfloor\sqrt{n}\rfloor-1} V_{k 3}, \\
& A^{(3)}=\frac{1}{n-3} \sum_{k=n-\lfloor\sqrt{n}\rfloor}^{n-2} V_{k 1}, \quad B^{(3)}=\frac{1}{n-3} \sum_{k=n-\lfloor\sqrt{n}\rfloor}^{n-2} V_{k 2}, \quad C^{(3)}=\frac{1}{n-3} \sum_{k=n-\lfloor\sqrt{n}\rfloor}^{n-2} V_{k 3} .
\end{aligned}
$$

Without loss of generality, we can assume that $\sigma_{1}(a, b)=\sigma_{2}(a, b)=0$. Observing the decomposition (5.1) the assertion (3.7) follows from

$$
\mathbb{P}\left\{\bigcup_{(a, b) \in \mathcal{N}}\left\{D^{(i)}(a, b)>c \tau\right\}\right\} \rightarrow 0, \quad i=1,2,3 .
$$

When we prove these results we derive exponential inequalities for all three probabilities, which directly yield the estimate in (3.6).

In (5.6) and hereafter in the proof, $c$ and $c_{i}(i=1,2, \cdots)$ indicate some positive constants that may change from line to line. According to the decomposition (5.4), it is sufficient to derive exponential inequalities, which will be used to verify the following results:

$$
p^{2} \cdot \mathbb{P}\left\{A^{(i)}>c \tau\right\} \rightarrow 0, \quad p^{2} \cdot \mathbb{P}\left\{B^{(i)}>c \tau\right\} \rightarrow 0, \quad p^{2} \cdot \mathbb{P}\left\{C^{(i)}>c \tau\right\} \rightarrow 0, \quad i=1,2,3 .
$$

The case $i=1,3$. For the index $i=1$ and $i=3$ the arguments are very similar and for the sake of brevity we only consider the case $i=1$ in (5.7). For the statistic $A^{(1)}$ we find that

$$
\begin{aligned}
\mathbb{P}\left\{A^{(1)}>c \tau\right\} & =\mathbb{P}\left\{\frac{1}{\sqrt{n}-1} \sum_{k=2}^{\lfloor\sqrt{n}\rfloor} \frac{\sqrt{n}-1}{n-3} \frac{k(n-k)}{n}\left[\dot{\bar{X}}_{k(a, b)}-\dot{\bar{Y}}_{k(a, b)}\right]^{2}>c \tau\right\} \\
& \leq \sum_{k=2}^{\lfloor\sqrt{n}\rfloor} \mathbb{P}\left\{\frac{\sqrt{n}-1}{n-3} \frac{k(n-k)}{n}\left[\dot{\bar{X}}_{k(a, b)}-\dot{\bar{Y}}_{k(a, b)}\right]^{2}>c \tau\right\} \\
& \leq \sum_{k=2}^{\lfloor\sqrt{n}\rfloor} \mathbb{P}\left\{\left|\dot{\bar{X}}_{k(a, b)}-\dot{\bar{Y}}_{k(a, b)}\right|>\sqrt{c \tau M_{n, k, \alpha}}\right\}
\end{aligned}
$$

where we use the notation $M_{n, k, \alpha}=\frac{\sqrt{n} \cdot n}{k(n-k)}$ for the sake of a transparent notation. Considering the components that constitute $\dot{\bar{X}}_{k(a, b)}$ and $\dot{\bar{Y}}_{k(a, b)}$ in (5.3), we use Lemma 5.1 and 5.2 to 
calculate the following probabilities to get an upper bound of (5.8)

$$
\begin{aligned}
\mathbb{P}\left\{\left|\bar{X}_{k(a, b)}\right|>\sqrt{c \tau M_{n, k, \alpha}}\right\} & \leq c_{1} e^{-c_{2} k \min \left(\tau M_{n, k, \alpha}, \sqrt{\tau M_{n, k, \alpha}}\right)} \\
& \leq c_{1} e^{-c_{2} \min (\tau \sqrt{n}, \sqrt{\tau \sqrt{n}})}, \\
\mathbb{P}\left\{\left|\bar{Y}_{k(a, b)}\right|>\sqrt{c \tau M_{n, k, \alpha}}\right\} & \leq c_{1} e^{-c_{2}(n-k) \min \left(\tau M_{n, k, \alpha}, \sqrt{\tau M_{n, k, \alpha}}\right)} \\
& \leq c_{1} e^{-c_{2} \min (\tau n, \sqrt{\tau n(n-\sqrt{n})})}
\end{aligned}
$$

$(k=2, \ldots,\lfloor\sqrt{n}\rfloor)$. Similarly, it follows from Lemma 5.3

$$
\begin{aligned}
& \mathbb{P}\left\{\left|\bar{X}_{k a} \bar{X}_{b}\right|>\sqrt{c \tau M_{n, k, \alpha}}\right\} \\
\leq & \mathbb{P}\left\{\left|\bar{X}_{k a}\right|>c_{1}\left(\tau M_{n, k, \alpha}\right)^{1 / 4}\right\}+\mathbb{P}\left\{\left|\bar{X}_{b}\right|>c_{2}\left(\tau M_{n, k, \alpha}\right)^{1 / 4}\right\} \\
\leq & c_{3}\left[e^{-c_{4} k \sqrt{\tau M_{n, k, \alpha}}}+e^{-c_{5} n \sqrt{\tau M_{n, k, \alpha}}}\right] \leq c_{3}\left[e^{-c_{4} \sqrt{\tau \sqrt{n}}}+e^{-c_{5} \sqrt{\tau} n}\right],
\end{aligned}
$$

for $k=2, \ldots,\lfloor\sqrt{n}\rfloor$, and using similar arguments we obtain for

$$
\begin{aligned}
& \mathbb{P}\left\{\left|\bar{Y}_{k a} \bar{X}_{b}\right|>\sqrt{c \tau M_{n, k, \alpha}}\right\} \leq c_{3}\left[e^{-c_{4} \sqrt{\tau n(n-\sqrt{n})}}+e^{-c_{5} \sqrt{\tau} n}\right], \\
& \mathbb{P}\left\{\left|\bar{X}_{a} \bar{X}_{b}\right|>\sqrt{c \tau M_{n, k, \alpha}}\right\} \leq c_{3} e^{-c_{4} n \sqrt{\tau M_{n, k, \alpha}}} \leq c_{3} e^{-c_{4} \sqrt{\tau} n},
\end{aligned}
$$

$k=2, \ldots\lfloor\sqrt{n}\rfloor$. The same estimates (with different constants $c, c_{1}, c_{2}, \ldots$ ) can be derived for the remaining terms involving $\bar{X}_{k b} \bar{X}_{a}$ and $\bar{Y}_{k b} \bar{X}_{a}$. Combining (5.3), (5.8)-(5.12) we obtain the upper bound

$$
\begin{aligned}
\mathbb{P}\left\{A^{(1)}>c \tau\right\} \leq & c_{1} \sqrt{n}\left(e^{-c_{2} \min (\tau \sqrt{n}, \sqrt{\tau \sqrt{n}})}+e^{-c_{2} \min (\tau n, \sqrt{\tau n(n-\sqrt{n})})}\right. \\
& \left.+e^{-c_{2} \sqrt{\tau \sqrt{n}}}+e^{-c_{2} \sqrt{\tau n(n-\sqrt{n})}}+e^{-c_{2} \sqrt{\tau} n}\right) .
\end{aligned}
$$

Since the smallest absolute value among the exponents in (5.13) is $\sqrt{\tau \sqrt{n}}$ we have

$$
\mathbb{P}\left\{A^{(1)}>c \tau\right\} \leq c_{3} \cdot \sqrt{n} e^{-c \sqrt{\tau} n^{1 / 4}}
$$

for some small positive constant $c$. Consequently, using the assumption (3.4) it follows that $p^{2} \cdot \mathbb{P}\left\{A^{(1)}>c \tau\right\} \rightarrow 0$.

Using similar arguments, we can investigate the other terms. To be precise consider the statistic $B^{(1)}$, for which we obtain the estimate

$$
\begin{aligned}
\mathbb{P}\left\{B^{(1)}>c \tau\right\} & =\mathbb{P}\left\{\frac{1}{n-3} \sum_{k=2}^{\lfloor\sqrt{n}\rfloor} \frac{k(n-k)}{n}\left[\frac{1}{k-1} \dot{\bar{X}}_{k(a, b)}^{2}+\frac{1}{n-k-1} \dot{\bar{Y}}_{k(a, b)}^{2}\right]>c \tau\right\} \\
& \leq \sum_{k=2}^{\lfloor\sqrt{n}\rfloor} \mathbb{P}\left\{\frac{\sqrt{n}-1}{n-3} \frac{k(n-k)}{n}\left[\frac{1}{k-1} \dot{\bar{X}}_{k(a, b)}^{2}+\frac{1}{n-k-1} \dot{\bar{Y}}_{k(a, b)}^{2}\right]>c \tau\right\} \\
& \leq \sum_{k=2}^{\lfloor\sqrt{n}\rfloor}\left[\mathbb{P}\left\{\left|\dot{\bar{X}}_{k(a, b)}\right|>\sqrt{c \tau M_{n, k, \alpha}^{(1)}}\right\}+\mathbb{P}\left\{\left|\dot{\bar{Y}}_{k(a, b)}\right|>\sqrt{c \tau M_{n, k, \alpha}^{(2)}}\right\}\right],
\end{aligned}
$$


where the quantities $M_{n, k, \alpha}^{(1)}$ and $M_{n, k, \alpha}^{(2)}$ are defined by

$$
M_{n, k, \alpha}^{(1)}=\frac{\sqrt{n} \cdot n}{(n-k)} \text { and } M_{n, k, \alpha}^{(2)}=\frac{\sqrt{n} \cdot n}{k},
$$

respectively. Again we consider the components that constitute $\dot{\bar{X}}_{k(a, b)}$ and $\dot{\bar{Y}}_{k(a, b)}$ in $(5.3)$ separately and obtain by an application of Lemma 5.1 and 5.2 the following estimates (for $k=2, \ldots,\lfloor\sqrt{n}\rfloor)$

$$
\begin{aligned}
\mathbb{P}\left\{\left|\bar{X}_{k(a, b)}\right|>\sqrt{c \tau M_{n, k, \alpha}^{(1)}}\right\} & \leq c_{1} e^{-c_{2} k \min \left(\tau M_{n, k, \alpha}^{(1)} \sqrt{\tau M_{n, k, \alpha}^{(1)}}\right)} \leq c_{1} e^{-c_{2} \min (\tau \sqrt{n}, \sqrt{\tau \sqrt{n}})}, \\
\mathbb{P}\left\{\left|\bar{Y}_{k(a, b)}\right|>\sqrt{c \tau M_{n, k, \alpha}^{(2)}}\right\} & \leq c_{1} e^{-c_{2}(n-k) \min \left(\tau M_{n, k, \alpha}^{(2)}, \sqrt{\tau M_{n, k, \alpha}^{(2)}}\right)} \\
& \leq c_{1} e^{-c_{2} \min \left(\tau n(n-\sqrt{n}), \sqrt{\tau n(n-\sqrt{n})^{2}}\right)} .
\end{aligned}
$$

Similarly, Lemma 5.3 gives for $k=2, \ldots,\lfloor\sqrt{n}\rfloor$

$$
\begin{aligned}
& \mathbb{P}\left\{\left|\bar{X}_{k a} \bar{X}_{b}\right|>\sqrt{c \tau M_{n, k, \alpha}^{(1)}}\right\} \leq c_{3}\left[e^{-c_{4} k \sqrt{\tau M_{n, k, \alpha}^{(1)}}}+e^{-c_{5} n \sqrt{\tau M_{n, k, \alpha}^{(1)}}}\right] \\
& \leq c_{3}\left[e^{-c_{4} \sqrt{\tau \sqrt{n}}}+e^{-c_{5} n \sqrt{\tau \sqrt{n}}}\right] \text {, } \\
& \mathbb{P}\left\{\left|\bar{Y}_{k a} \bar{X}_{b}\right|>\sqrt{c \tau M_{n, k, \alpha}^{(2)}}\right\} \leq c_{3}\left[e^{-c_{4}(n-k) \sqrt{\tau M_{n, k, \alpha}^{(2)}}}+e^{-c_{5} n \sqrt{\tau M_{n, k, \alpha}^{(2)}}}\right] \\
& \leq c_{3}\left[e^{-c_{4}(n-\sqrt{n}) \sqrt{\tau n}}+e^{-c_{5} n \sqrt{\tau n}}\right] \text {, } \\
& \mathbb{P}\left\{\left|\bar{X}_{a} \bar{X}_{b}\right|>\sqrt{c \tau M_{n, k, \alpha}^{(1)}}\right\} \leq c_{3} e^{-c_{4} n \sqrt{\tau M_{n, k, \alpha}^{(1)}}} \leq c_{3} e^{-c_{4} n \sqrt{\tau \sqrt{n}}}, \\
& \mathbb{P}\left\{\left|\bar{X}_{a} \bar{X}_{b}\right|>\sqrt{c \tau M_{n, k, \alpha}^{(2)}}\right\} \leq c_{3} e^{-c_{4} n \sqrt{\tau M_{n, k, \alpha}^{(2)}}} \leq c_{3} e^{-c_{4} n \sqrt{\tau n}} .
\end{aligned}
$$

Thus, observing that the smallest absolute value among the exponents in these estimates is given by $\sqrt{\tau \sqrt{n}}$, an upper bound for the probability in (5.14) is obtained as

$$
\mathbb{P}\left\{B^{(1)}>c \tau\right\} \leq c_{1} \sqrt{n} e^{-c \sqrt{\tau} n^{1 / 4}}
$$

for some small positive constant $c$. Consequently, observing Assumption 3.3 we have $p^{2} \mathbb{P}\left\{B^{(1)}>\right.$ $c \tau\} \rightarrow 0$ as $n, p \rightarrow \infty$.

Finally the term $C^{(1)}$ is estimated as follows

$$
\begin{aligned}
\mathbb{P}\left\{C^{(1)}>c \tau\right\} & \leq \sum_{k=2}^{\lfloor\sqrt{n}\rfloor} \mathbb{P}\left\{\frac{\sqrt{n}-1}{n-3} \frac{k(n-k)}{n}\left[\frac{1}{k(k-1)} \sum_{i=1}^{k}\left(\dot{X}_{i a} \dot{X}_{i b}\right)^{2}+\frac{1}{(n-k)(n-k-1)} \sum_{i=k+1}^{n}\left(\dot{X}_{i a} \dot{X}_{i b}\right)^{2}\right]>c \tau\right\} \\
& \leq \sum_{k=2}^{\lfloor\sqrt{n}\rfloor}\left[\mathbb{P}\left\{\frac{1}{k} \sum_{i=1}^{k}\left(\dot{X}_{i a} \dot{X}_{i b}\right)^{2}>c_{1} \tau \frac{n \sqrt{n}}{n-k}\right\}+\mathbb{P}\left\{\frac{1}{n-k} \sum_{i=k+1}^{n}\left(\dot{X}_{i a} \dot{X}_{i b}\right)^{2}>c_{2} \tau \frac{n \sqrt{n}}{k}\right\}\right] \\
& \leq \sum_{k=2}^{\lfloor\sqrt{n}\rfloor}\left[k \mathbb{P}\left\{\left|\dot{X}_{i a} \dot{X}_{i b}\right|>\sqrt{c_{1} \tau \sqrt{n}}\right\}+(n-k) \mathbb{P}\left\{\left|\dot{X}_{i a} \dot{X}_{i b}\right|>\sqrt{c_{2} \tau n}\right\}\right] .
\end{aligned}
$$


Observing that $\dot{X}_{i a} \dot{X}_{i b}=X_{i a} X_{i b}-X_{i a} \bar{X}_{b}-X_{i b} \bar{X}_{a}+\bar{X}_{a} \bar{X}_{b}$, we obtain from Lemma 5.1 and 5.3 the estimates

$$
\begin{aligned}
k \mathbb{P}\left\{\left|X_{i a} X_{i b}\right|>\sqrt{c_{1} \tau \sqrt{n}}\right\} & \leq c \sqrt{n} e^{-c_{4} \sqrt{\tau \sqrt{n}}} \\
(n-k) \mathbb{P}\left\{\left|X_{i a} X_{i b}\right|>\sqrt{c_{2} \tau n}\right\} & \leq c n e^{-c_{6} \sqrt{\tau n}} \\
k \mathbb{P}\left\{\left|X_{i a} \bar{X}_{b}\right|>\sqrt{c_{1} \tau \sqrt{n}}\right\} & \leq c \sqrt{n}\left[e^{-c_{7} \sqrt{\tau \sqrt{n}}}+e^{-c_{8} n \sqrt{\tau \sqrt{n}}}\right], \\
(n-k) \mathbb{P}\left\{\left|X_{i a} \bar{X}_{b}\right|>\sqrt{c_{2} \tau n}\right\} & \leq c n\left[e^{-c_{7} \sqrt{\tau n}}+e^{-c_{8} n \sqrt{\tau n}}\right], \\
k \mathbb{P}\left\{\left|\bar{X}_{a} \bar{X}_{b}\right|>\sqrt{c_{1} \tau \sqrt{n}}\right\} & \leq c \sqrt{n} e^{-c_{7} n \sqrt{\tau \sqrt{n}}}, \\
(n-k) \mathbb{P}\left\{\left|\bar{X}_{a} \bar{X}_{b}\right|>\sqrt{c_{2} \tau n}\right\} & \leq c n e^{-c_{7} n \sqrt{\tau n}},
\end{aligned}
$$

whenever $k=2, \ldots,\lfloor\sqrt{n}\rfloor$. Summarizing we have

$$
\begin{aligned}
\mathbb{P}\left\{C^{(1)}>c \tau\right\} & \leq c_{1}\left[n\left(e^{-c_{2} \sqrt{\tau \sqrt{n}}}+e^{-c_{2} n \sqrt{\tau \sqrt{n}}}\right)+n \sqrt{n}\left(e^{-c_{2} \sqrt{\tau n}}+e^{-c_{2} n \sqrt{\tau n}}\right)\right] \\
& \leq c_{3} n e^{-c \sqrt{\tau \sqrt{n}}}
\end{aligned}
$$

for some small positive constant $c$. Now assumption (3.4) implies $p^{2} \mathbb{P}\left\{C^{(1)}>c \tau\right\} \rightarrow 0$, if

$$
p^{2} n e^{-c \sqrt{\tau} n^{1 / 4}} \rightarrow 0
$$

and therefore the proof of (5.7) in the case $i=1$ is completed (the case $i=3$ follows by exactly the same arguments).

The case $i=2$. For the term $A^{(2)}$, we get that

$$
\mathbb{P}\left\{A^{(2)}>c \tau\right\} \leq \sum_{k=\lfloor\sqrt{n}\rfloor+1}^{n-\lfloor\sqrt{n}\rfloor-1}\left[\mathbb{P}\left\{\left|\dot{\bar{X}}_{k(a, b)}\right|>\sqrt{c_{1} \tau M_{n, k}}\right\}+\mathbb{P}\left\{\left|\dot{\bar{Y}}_{k(a, b)}\right|>\sqrt{c_{2} \tau M_{n, k}}\right\}\right]
$$

where we use the notation $M_{n, k}=\frac{n}{k(n-k)}$. Similar calculations as given for the term $A^{(1)}$ give for the summands in the representation (5.3) of $\dot{\bar{X}}_{k(a, b)}$ and $\dot{\bar{Y}}_{k(a, b)}$ the estimates (here we use the fact that for $i=2$ we have $k=\lfloor\sqrt{n}\rfloor+1, \ldots, n-\lfloor\sqrt{n}\rfloor-1$ )

$$
\begin{aligned}
& \mathbb{P}\left\{\left|\bar{X}_{k(a, b)}\right|>\sqrt{c \tau M_{n, k}}\right\} \leq c_{1} e^{-c_{2} k \min \left(\tau M_{n, k}, \sqrt{\tau M_{n, k}}\right)} \leq c_{1} e^{-c_{2} \min (\tau, \sqrt{\tau \sqrt{n}})}, \\
& \mathbb{P}\left\{\left|\bar{Y}_{k(a, b)}\right|>\sqrt{c \tau M_{n, k}}\right\} \leq c_{1} e^{-c_{2}(n-k) \min \left(\tau M_{n, k}, \sqrt{\tau M_{n, k}}\right)} \leq c_{1} e^{-c_{2} \min (\tau, \sqrt{\tau \sqrt{n}})}, \\
& \mathbb{P}\left\{\left|\bar{X}_{k a} \bar{X}_{b}\right|>\sqrt{c \tau M_{n, k}}\right\} \leq c_{3}\left[e^{-c_{4} k \sqrt{\tau M_{n, k}}}+e^{-c_{5} n \sqrt{\tau M_{n, k}}}\right] \leq c_{3}\left[e^{-c_{4} \sqrt{\tau \sqrt{n}}}+e^{-c_{5} \sqrt{\tau n}}\right], \\
& \mathbb{P}\left\{\left|\bar{Y}_{k a} \bar{X}_{b}\right|>\sqrt{c \tau M_{n, k}}\right\} \leq c_{3}\left[e^{-c_{4}(n-k) \sqrt{\tau M_{n, k}}}+e^{-c_{5} n \sqrt{\tau M_{n, k}}}\right] \leq c_{3}\left[e^{-c_{4} \sqrt{\tau \sqrt{n}}}+e^{-c_{5} \sqrt{\tau n}}\right], \\
& \mathbb{P}\left\{\left|\bar{X}_{a} \bar{X}_{b}\right|>\sqrt{c \tau M_{n, k}}\right\} \leq c_{3} e^{-c_{4} n \sqrt{\tau M_{n, k}}} \leq c_{3} e^{-c_{4} \sqrt{\tau n}},
\end{aligned}
$$


and consequently we obtain

$$
p^{2} \cdot \mathbb{P}\left\{A^{(2)}>c \tau\right\} \leq c_{1} n p^{2} \max \left\{e^{-c \tau}, e^{-c \sqrt{\tau \sqrt{n}}}\right\}
$$

for some small positive constant $c$, which converges to 0 under the stated assumptions (3.3) and (3.4).

For the term $B^{(2)}$ we have

$$
\mathbb{P}\left\{B^{(2)}>c \tau\right\} \leq \sum_{k=\lfloor\sqrt{n}\rfloor+1}^{n-\lfloor\sqrt{n}\rfloor-1}\left[\mathbb{P}\left\{\left|\dot{\bar{X}}_{k(a, b)}\right|>\sqrt{c_{1} \tau \frac{n}{n-k}}\right\}+\mathbb{P}\left\{\left|\dot{\bar{Y}}_{k(a, b)}\right|>\sqrt{c_{2} \tau \frac{n}{k}}\right\}\right]
$$

where the two probabilities can be bounded taking into account the representation (5.3) and the estimates

$$
\begin{aligned}
& \mathbb{P}\left\{\left|\bar{X}_{k(a, b)}\right|>\sqrt{\frac{c_{1} \tau n}{n-k}}\right\} \leq c_{1} e^{-c_{2} k \min \left(\frac{\tau n}{n-k}, \sqrt{\frac{\tau n}{n-k}}\right)} \leq c_{1} e^{-c_{2} \min (\tau \sqrt{n}, \sqrt{\tau n})}, \\
& \mathbb{P}\left\{\left|\bar{Y}_{k(a, b)}\right|>\sqrt{\frac{c \tau n}{k}}\right\} \leq c_{1} e^{-c_{2}(n-k) \min \left(\frac{\tau n}{k}, \sqrt{\frac{\tau n}{k}}\right)} \leq c_{1} e^{-c_{2} \min (\tau \sqrt{n}, \sqrt{\tau n})}, \\
& \mathbb{P}\left\{\left|\bar{X}_{k a} \bar{X}_{b}\right|>\sqrt{\frac{c \tau n}{n-k}}\right\} \leq c_{3}\left[e^{-c_{4} k \sqrt{\frac{\tau n}{n-k}}}+e^{-c_{5} n \sqrt{\frac{\tau n}{n-k}}}\right] \leq c_{3}\left[e^{-c_{4} \sqrt{\tau n}}+e^{-c_{5} n \sqrt{\tau}}\right], \\
& \mathbb{P}\left\{\left|\bar{Y}_{k a} \bar{X}_{b}\right|>\sqrt{\frac{c \tau n}{k}}\right\} \leq c_{3}\left[e^{-c_{4}(n-k) \sqrt{\frac{\tau n}{k}}}+e^{-c_{5} n \sqrt{\frac{\tau n}{k}}}\right] \leq c_{3}\left[e^{-c_{4} \sqrt{\tau n}}+e^{-c_{5} n \sqrt{\tau}}\right], \\
& \mathbb{P}\left\{\left|\bar{X}_{a} \bar{X}_{b}\right|>\sqrt{\frac{c \tau n}{n-k}}\right\} \leq c_{3} e^{-c_{4} n \sqrt{\frac{\tau n}{n-k}}} \leq c_{3} e^{-c_{4} n \sqrt{\tau}}, \\
& \mathbb{P}\left\{\left|\bar{X}_{a} \bar{X}_{b}\right|>\sqrt{\frac{c \tau n}{k}}\right\} \leq c_{3} e^{-c_{4} n \sqrt{\tau}}
\end{aligned}
$$

for $k=\lfloor\sqrt{n}\rfloor+1,\lfloor\sqrt{n}\rfloor+2, \ldots, n-\lfloor\sqrt{n}\rfloor-1$. Therefore, we obtain as an upper bound for the probability in $(5.16)$

$$
\begin{aligned}
p^{2} \cdot \mathbb{P}\left\{B^{(2)}>c \tau\right\} & \leq c_{1} p^{2} n\left(e^{-c_{2} \min (\tau \sqrt{n}, \sqrt{\tau n})}+e^{-c_{2} \sqrt{\tau n}}+e^{-c_{2} n \sqrt{\tau}}\right) \\
& =c_{1} p^{2} n\left(e^{-c_{2} \sqrt{n} \sqrt{\tau}}+e^{-c_{2} \sqrt{\tau n}}+e^{-c_{2} n \sqrt{\tau}}\right)=o(1)
\end{aligned}
$$

by assumption (3.4).

For the term $C^{(2)}$, we observe (note that $n /(n-k)>1$ and $n / k>1$ )

$$
\begin{aligned}
\mathbb{P}\left\{C^{(2)}>c \tau\right\} & \leq \sum_{k=\lfloor\sqrt{n}\rfloor+1}^{n-\lfloor\sqrt{n}\rfloor-1}\left[\mathbb{P}\left\{\frac{1}{k} \sum_{i=1}^{k}\left(\dot{X}_{i a} \dot{X}_{i b}\right)^{2}>c_{1} \tau \frac{n}{n-k}\right\}+\mathbb{P}\left\{\frac{1}{n-k} \sum_{i=k+1}^{n}\left(\dot{X}_{i a} \dot{X}_{i b}\right)^{2}>c_{2} \tau \frac{n}{k}\right\}\right] \\
& \leq \sum_{k=\lfloor\sqrt{n}\rfloor+1}^{n-\lfloor\sqrt{n}\rfloor-1} k \mathbb{P}\left\{\left|\dot{X}_{i a} \dot{X}_{i b}\right|>\sqrt{c_{1} \tau}\right\} \\
& +\sum_{k=\lfloor\sqrt{n}\rfloor+1}^{n-\lfloor\sqrt{n}\rfloor-1}(n-k) \mathbb{P}\left\{\left|\dot{X}_{i a} \dot{X}_{i b}\right|>\sqrt{c_{2} \tau}\right\},
\end{aligned}
$$


where the two probabilities can be bounded using the representation $\dot{X}_{i a} \dot{X}_{i b}=X_{i a} X_{i b}-$ $X_{i a} \bar{X}_{b}-X_{i b} \bar{X}_{a}+\bar{X}_{a} \bar{X}_{b}$. This gives for $k=\lfloor\sqrt{n}\rfloor+1, \ldots, n-\lfloor\sqrt{n}\rfloor-1$ the estimates

$$
\begin{aligned}
& \mathbb{P}\left\{\left|X_{i a} X_{i b}\right|>c \sqrt{\tau}\right\} \leq c_{1} e^{-c_{2} \sqrt{\tau}}, \\
& \mathbb{P}\left\{\left|X_{i a} \bar{X}_{b}\right|>c \sqrt{\tau}\right\} \leq\left[\mathbb{P}\left\{\left|X_{i a}\right|>c_{1} \tau^{1 / 4}\right\}+\mathbb{P}\left\{\left|\bar{X}_{b}\right|>c_{2} \tau^{1 / 4}\right\}\right] \leq c_{3}\left[e^{-c_{4} \sqrt{\tau}}+e^{-c_{5} n \sqrt{\tau}}\right], \\
& \mathbb{P}\left\{\left|\bar{X}_{a} \bar{X}_{b}\right|>c \sqrt{\tau}\right\} \leq\left[\mathbb{P}\left\{\left|\bar{X}_{a}\right|>c_{1} \tau^{1 / 4}\right\}+\mathbb{P}\left\{\left|\bar{X}_{b}\right|>c_{2} \tau^{1 / 4}\right\}\right] \leq c_{3} e^{-c_{4} n \sqrt{\tau}} .
\end{aligned}
$$

Observing that $\sum_{k=\lfloor\sqrt{n}\rfloor+1}^{n-\lfloor\sqrt{n}\rfloor-1} k \leq n^{2}, \sum_{k=\lfloor\sqrt{n}\rfloor+1}^{n-\lfloor\sqrt{n}\rfloor-1}(n-k) \leq n^{2}$ we can bound the probability on the left hand side of (5.16) by

$$
\mathbb{P}\left\{C^{(2)}>c \tau\right\} \leq c_{1} n^{2}\left(e^{-c_{2} \sqrt{\tau}}+e^{-c_{3} n \sqrt{\tau}}\right),
$$

which vanishes asymptotically by assumption (3.5).

Therefore, we have established (5.7) for all indices $i=1,2,3$ which implies (5.6), and the assertion of Theorem 3.1 follows. A careful inspection of our arguments shows that we have also established the estimate (3.6) in Theorem 3.1.

\subsection{Proof of Theorem 3.2}

Recall that the set $\mathcal{P}$ is the set of indices corresponding to elements with $\left|\sigma_{1}(a, b)-\sigma_{2}(a, b)\right|>$ $\lambda$, where $\lambda$ satisfies (3.2), and note that the assertion (3.9) is equivalent to

$$
\mathbb{P}\left\{\bigcup_{(a, b) \in \mathcal{P}}\{D(a, b) \leq \tau\}\right\} \rightarrow 0
$$

For a proof of this statement we derive several exponential inequalities which directly yield the estimate in (3.8). For this purpose we introduce the decomposition

$$
D(a, b)=\frac{1}{n-3} \sum_{k=2}^{n-2}\left[V_{k 1}+V_{k 2}-V_{k 3}\right]=A+B-C,
$$

where the quantities $A, B$ and $C$ are given by

$$
A=\frac{1}{n-3} \sum_{k=2}^{n-2} V_{k 1}, \quad B=\frac{1}{n-3} \sum_{k=2}^{n-2} V_{k 2}, \quad C=\frac{1}{n-3} \sum_{k=2}^{n-2} V_{k 3},
$$

respectively, and the statistics $V_{k 1}, V_{k 2}$ and $V_{k_{3}}$ have been defined in (5.2). Observing the inclusion

$$
\{D(a, b) \leq \tau\} \subset\{A \leq 3 \tau\} \cup\{|B-C| \geq 2 \tau\} \subset\{A \leq 3 \tau\} \cup\{B \geq \tau\} \cup\{C \geq \tau\}
$$


the assertion of Theorem 3.2 follows by deriving exponential inequalities for the probabilities for these three events, which are used to prove

$$
p^{2} \cdot \mathbb{P}\{A \leq 3 \tau\} \rightarrow 0, \quad p^{2} \cdot \mathbb{P}\{B \geq \tau\} \rightarrow 0, \quad p^{2} \cdot \mathbb{P}\{C \geq \tau\} \rightarrow 0
$$

We now investigate the three probabilities in (5.18) separately. First, for the last two terms $B$ and $C$, from (5.5), it is easy to see that $B=B^{(1)}+B^{(2)}+B^{(3)}$ and $C=C^{(1)}+C^{(2)}+C^{(3)}$, where - as in the proof of Theorem 3.1 - the indices (1), (2) and (3) correspond to the summation with respect to the sets $\{2, \ldots,\lfloor\sqrt{n}\rfloor\},\{\lfloor\sqrt{n}\rfloor+1, \ldots, n-\lfloor\sqrt{n}\rfloor-1\}$ and $\{n-\lfloor\sqrt{n}\rfloor, \ldots, n-2\}$, respectively. Moreover, using similar arguments as given in the proof of Theorem 3.1, the following results can be established.

$$
\begin{aligned}
& p^{2} \cdot \mathbb{P}\left\{B^{(i)}>c \tau\right\} \leq c_{1} p^{2} \sqrt{n} e^{-c \sqrt{\tau} n^{1 / 4}}, i=1,3, \quad p^{2} \cdot \mathbb{P}\left\{B^{(2)}>c \tau\right\} \leq c_{1} p^{2} n e^{-c_{2} \sqrt{\tau n}}, \\
& p^{2} \cdot \mathbb{P}\left\{C^{(i)}>c \tau\right\} \leq c_{3} p^{2} n e^{-c \sqrt{\tau} n^{1 / 4}}, i=1,3, \quad p^{2} \cdot \mathbb{P}\left\{C^{(2)}>c \tau\right\} \leq c_{1} p^{2} n^{2} e^{-c_{2} \sqrt{\tau}} .
\end{aligned}
$$

The only difference when analyzing the above probabilities lies in the expectation of $X_{i a} X_{i b}$, $\mathbb{E}\left(X_{i a} X_{i b}\right)$, which is not necessarily zero now. But this does not affect the proof of (5.19) since $\mathbb{E}\left(X_{i a} X_{i b}\right)$ is always bounded by Assumption 3.1. Hence

$$
p^{2} \cdot \mathbb{P}\{B \geq \tau\} \rightarrow 0, \quad p^{2} \cdot \mathbb{P}\{C \geq \tau\} \rightarrow 0
$$

In order to show the remaining exponential equation and prove the assertion $p^{2} \cdot \mathbb{P}\{A \leq$ $3 \tau\} \rightarrow 0$, we note that $\sigma_{1}(a, b) \neq \sigma_{2}(a, b)$, and a straightforward calculation gives

$$
\mathbb{E}\left(\bar{X}_{k(a, b)}-\bar{Y}_{k(a, b)}\right)= \begin{cases}\frac{n-k_{0}}{n-k}\left(\sigma_{1}(a, b)-\sigma_{2}(a, b)\right) & : k \leq k_{0}, \\ \frac{k_{0}}{k}\left(\sigma_{1}(a, b)-\sigma_{2}(a, b)\right) & : k>k_{0}\end{cases}
$$

Let

$$
A_{k}=\dot{\bar{X}}_{k(a, b)}-\dot{\bar{Y}}_{k(a, b)}-\mathbb{E}\left(\bar{X}_{k(a, b)}-\bar{Y}_{k(a, b)}\right), \quad B_{k}=\mathbb{E}\left(\bar{X}_{k(a, b)}-\bar{Y}_{k(a, b)}\right),
$$

and define $b_{n, k} \triangleq \frac{k(n-k)}{n}$. Then one can observe that

$$
\left|B_{k}\right| \geq \min \left\{\frac{n-k_{0}}{n}, \frac{k_{0}}{n}\right\} \cdot\left|\sigma_{1}(a, b)-\sigma_{2}(a, b)\right|
$$

and

$$
\begin{aligned}
\frac{1}{n-3} \sum_{k=2}^{n-2} b_{n, k} B_{k}^{2} & \geq \min \left\{\frac{\left(n-k_{0}\right)^{2}}{n^{2}}, \frac{k_{0}^{2}}{n^{2}}\right\} \cdot n\left(\sigma_{1}(a, b)-\sigma_{2}(a, b)\right)^{2} \cdot \frac{1}{n-3} \sum_{k=2}^{n-2} \frac{k(n-k)}{n^{2}} \\
& \geq \min \left\{\frac{\left(n-k_{0}\right)^{2}}{n^{2}}, \frac{k_{0}^{2}}{n^{2}}\right\} \cdot \frac{n\left(\sigma_{1}(a, b)-\sigma_{2}(a, b)\right)^{2}}{6} \geq \min \left\{\frac{\left(n-k_{0}\right)^{2}}{n^{2}}, \frac{k_{0}^{2}}{n^{2}}\right\} \cdot \frac{n \lambda^{2}}{6} .
\end{aligned}
$$


Consequently, by assumption (3.2) (with a sufficiently large constant $C$ ) we obtain the estimate

$$
3 \tau \leq \frac{1}{2(n-3)} \sum_{k=2}^{n-2} b_{n, k} B_{k}^{2} .
$$

On the other hand, we have by the definition of $A, A_{k}$ and $B_{k}$

$$
\begin{aligned}
\mathbb{P}\{A \leq 3 \tau\} & =\mathbb{P}\left\{\frac{1}{n-3} \sum_{k=2}^{n-2} b_{n, k}\left[A_{k}+B_{k}\right]^{2} \leq 3 \tau\right\} \\
& \leq \mathbb{P}\left\{\frac{2}{n-3} \sum_{k=2}^{n-2} b_{n, k} A_{k} B_{k} \leq 3 \tau-\frac{1}{n-3} \sum_{k=2}^{n-2} b_{n, k} B_{k}^{2}\right\} \\
& \leq \mathbb{P}\left\{\frac{2}{n-3} \sum_{k=2}^{n-2} b_{n, k} A_{k} B_{k} \leq-\frac{1}{2(n-3)} \sum_{k=2}^{n-2} b_{n, k} B_{k}^{2}\right\} \\
& \leq \mathbb{P}\left\{\frac{2}{n-3}\left|\sum_{k=2}^{n-2} b_{n, k} A_{k} B_{k}\right| \geq \frac{1}{2(n-3)}\left|\sum_{k=2}^{n-2} b_{n, k} B_{k}^{2}\right|\right\} \\
& \leq n \cdot \mathbb{P}\left\{\left|b_{n, k} A_{k}\right| \geq \frac{c}{\left|B_{k}\right|} \min \left\{\frac{\left(n-k_{0}\right)^{2}}{n^{2}}, \frac{k_{0}^{2}}{n^{2}}\right\} \cdot n\left(\sigma_{1}(a, b)-\sigma_{2}(a, b)\right)^{2}\right\} \\
& =n \cdot \mathbb{P}\left\{\left|A_{k}\right| \geq c \phi\right\},
\end{aligned}
$$

where $\phi=\frac{1}{\left|B_{k}\right|} \frac{1}{b_{n, k}} \min \left\{\frac{\left(n-k_{0}\right)^{2}}{n^{2}}, \frac{k_{0}^{2}}{n^{2}}\right\} \cdot n\left(\sigma_{1}(a, b)-\sigma_{2}(a, b)\right)^{2}$. In order to investigate the probability $\mathbb{P}\left\{\left|A_{k}\right| \geq c \phi\right\}$ in (5.21) note that $\frac{1}{\left|B_{k}\right|} \geq \frac{1}{\left|\sigma_{1}(a, b)-\sigma_{2}(a, b)\right|}$ by (5.20). If

$$
\left|\sigma_{1}(a, b)-\sigma_{2}(a, b)\right| \geq \lambda \geq C \sqrt{\frac{\tau}{n}} \max \left\{\frac{n^{2}}{\left(n-k_{0}\right)^{2}}, \frac{n^{2}}{k_{0}^{2}}\right\}
$$

it is therefore easy to see that

$$
\phi \geq \frac{n}{k(n-k)} \min \left\{\frac{\left(n-k_{0}\right)^{2}}{n^{2}}, \frac{k_{0}^{2}}{n^{2}}\right\} \cdot n\left|\sigma_{1}(a, b)-\sigma_{2}(a, b)\right| \geq C \frac{n}{k(n-k)} \sqrt{n \tau} .
$$

Observing the decomposition (5.3), the term $A_{k}$ can be written as

$$
A_{k}=\left[\bar{X}_{k(a, b)}-\bar{Y}_{k(a, b)}-\mathbb{E}\left(\bar{X}_{k(a, b)}-\bar{Y}_{k(a, b)}\right)\right]-\bar{X}_{k a} \bar{X}_{b}-\bar{X}_{k b} \bar{X}_{a}+\bar{Y}_{k a} \bar{X}_{b}+\bar{Y}_{k b} \bar{X}_{a},
$$

and by Lemma 5.2 and Lemma 5.3 we obtain

$$
\begin{aligned}
& \mathbb{P}\left\{\left|A_{k}\right| \geq c \phi\right\} \\
\leq & \mathbb{P}\left\{\left|\bar{X}_{k(a, b)}-\mathbb{E} \bar{X}_{k(a, b)}\right| \geq c_{1} \phi\right\}+\mathbb{P}\left\{\left|\bar{Y}_{k(a, b)}-\mathbb{E} \bar{Y}_{k(a, b)}\right| \geq c_{2} \phi\right\} \\
& +2 \mathbb{P}\left\{\left|\bar{X}_{k a}\right| \geq c_{3} \sqrt{\phi}\right\}+2 \mathbb{P}\left\{\left|\bar{X}_{b}\right| \geq c_{4} \sqrt{\phi}\right\}+2 \mathbb{P}\left\{\left|\bar{Y}_{k a}\right| \geq c_{3} \sqrt{\phi}\right\}+2 \mathbb{P}\left\{\left|\bar{X}_{b}\right| \geq c_{4} \sqrt{\phi}\right\} \\
\leq & c_{5}\left[e^{-c_{6} k \min \left\{\phi^{2}, \phi\right\}}+e^{-c_{6}(n-k) \min \left\{\phi^{2}, \phi\right\}}+e^{-c_{6} k \phi}+e^{-c_{6}(n-k) \phi}+e^{-c_{6} n \phi}\right] \\
\leq & c_{5}\left[e^{-c_{6} \min \{\tau, \sqrt{n \tau}\}}+e^{-c_{6} \min \{\tau, \sqrt{n \tau}\}}+e^{-c_{6} \sqrt{n \tau}}+e^{-c_{6} \sqrt{n \tau}}+e^{-c_{6} \sqrt{n \tau}}\right]=o(1),
\end{aligned}
$$

where the last estimate follows from (3.3) and (3.5). Combining (5.21) with (5.22), it follows that $p^{2} \cdot \mathbb{P}\{A \leq 3 \tau\} \rightarrow 0$, which completes the proof of Theorem 3.2. 


\subsection{Proof of Theorem 3.3}

Recall the definition of the statistic $U_{n}(k)$ in $(2.5)$ and the definition of the change point estimator $\hat{k}$ in (2.6). Let $\widetilde{\mathbf{x}}_{i}$ indicate the $m$-dimensional subvector of vech $\left(\mathbf{x}_{i} \mathbf{x}_{i}^{T}\right)$ corresponding to the components in the set $\mathcal{D}_{\tau}$ in (2.4). Obviously,

$$
\mathbb{P}\left\{\left|\frac{\hat{k}}{k_{0}}-1\right| \geq \epsilon\right\} \leq \mathbb{P}\left\{\hat{k} \geq(1+\epsilon) k_{0}\right\}+\mathbb{P}\left\{\hat{k} \leq(1-\epsilon) k_{0}\right\},
$$

and we will derive exponential bounds for the two terms on the right-hand side to prove that the probability vanishes asymptotically. We only consider the first term because the second term can be handled similarly. It is sufficient to show that

$$
P\left\{\bigcup_{k \geq(1+\epsilon) k_{0}}\left(U_{n}(k) \geq U_{n}\left(k_{0}\right)\right)\right\} \leq \sum_{k \geq(1+\epsilon) k_{0}}^{n} P\left\{U_{n}(k) \geq U_{n}\left(k_{0}\right)\right\} \leq c_{1} p^{2} n\left[e^{-c_{2} \tau}+n e^{-c_{2} \sqrt{\tau}}\right],
$$

which follows if the estimate

$$
P\left\{U_{n}(k) \geq U_{n}\left(k_{0}\right)\right\} \leq c_{1} p^{2}\left[e^{-c_{2} \tau}+n e^{-c_{2} \sqrt{\tau}}\right]
$$

holds. By Assumption 3.3 this term is of order $o\left(\frac{1}{n}\right)$ uniformly with respect to $(1+\varepsilon) k_{0} \leq$ $k \leq n$. For a proof of this statement define the vectors $\mathbf{w}_{i}=\left(W_{i 1}, \cdots, W_{i m}\right)$ by

$$
\dot{\widetilde{\mathbf{x}}}_{i}=\mathbf{w}_{i}+\mathbb{E} \widetilde{\mathbf{x}}_{i}
$$

and denote by $\boldsymbol{\mu}_{1}$ and $\boldsymbol{\mu}_{2}$ the $m$-dimensional vectors containing the elements of the matrices $\Sigma_{1}$ and $\Sigma_{2}$, respectively, which correspond to positions $(a, b) \in \mathcal{D}_{\tau}$ identified in Step 1 of the procedure. We will make use of the decomposition

$$
\begin{aligned}
U_{n}(k) & =\frac{1}{n^{4}} \sum_{(i \neq t)=1}^{k} \sum_{(j \neq l)=k+1}^{n} \sum^{n}\left[\left(\mathbf{w}_{i}-\mathbf{w}_{j}\right)+\left(\mathbb{E} \widetilde{\mathbf{x}}_{i}-\mathbb{E} \widetilde{\mathbf{x}}_{j}\right)\right]^{T}\left[\left(\mathbf{w}_{t}-\mathbf{w}_{l}\right)+\left(\mathbb{E} \widetilde{\mathbf{x}}_{t}-\mathbb{E} \widetilde{\mathbf{x}}_{l}\right)\right] \\
& =A(k)+B(k)+C(k)+E(k),
\end{aligned}
$$

where

$$
\begin{aligned}
& A(k)=\frac{1}{n^{4}} \sum_{(i \neq t)=1}^{k} \sum_{(j \neq l)=k+1}^{k} \sum_{k}^{n}\left(\mathbf{w}_{i}-\mathbf{w}_{j}\right)^{T}\left(\mathbf{w}_{t}-\mathbf{w}_{l}\right), \\
& B(k)=\frac{1}{n^{4}} \sum_{(i \neq t)=1}^{k} \sum_{(j \neq l)=k+1}^{n} \sum_{i}^{n}\left(\mathbb{E} \widetilde{\mathbf{x}}_{t}-\mathbb{E} \widetilde{\mathbf{x}}_{l}\right)^{T}\left(\mathbf{w}_{i}-\mathbf{w}_{j}\right), \\
& C(k)=\frac{1}{n^{4}} \sum_{(i \neq t)=1}^{k} \sum_{(j \neq l)=k+1}^{n} \sum_{n}^{n}\left(\mathbb{E} \widetilde{\mathbf{x}}_{i}-\mathbb{E} \widetilde{\mathbf{x}}_{j}\right)^{T}\left(\mathbf{w}_{t}-\mathbf{w}_{l}\right), \\
& E(k)=\frac{1}{n^{4}} \sum_{(i \neq t)=1}^{k} \sum_{(j \neq l)=k+1}^{k} \sum^{n}\left(\mathbb{E} \widetilde{\mathbf{x}}_{i}-\mathbb{E} \widetilde{\mathbf{x}}_{j}\right)^{T}\left(\mathbb{E} \widetilde{\mathbf{x}}_{t}-\mathbb{E} \widetilde{\mathbf{x}}_{l}\right),
\end{aligned}
$$


and begin investigating the constant terms $E(k)$ and $E\left(k_{0}\right)$. For this purpose we make use of the notation

$$
a_{n, k}=(n-k)(n-k-1)
$$

and obtain by a direct calculation

$$
\begin{gathered}
E=E\left(k_{0}\right)-E(k)=\frac{1}{n^{4}}\left[k_{0}\left(k_{0}-1\right) a_{n, k_{0}}-k_{0}\left(k_{0}-1\right) a_{n, k}\right]\left\|\boldsymbol{\mu}_{1}-\boldsymbol{\mu}_{2}\right\|^{2} \\
=\frac{1}{n^{4}} k_{0}\left(k_{0}-1\right)\left[(n-k)\left(k-k_{0}\right)+(n-k-1)\left(k-k_{0}\right)+\left(k-k_{0}\right)^{2}\right]\left\|\boldsymbol{\mu}_{1}-\boldsymbol{\mu}_{2}\right\|^{2} .
\end{gathered}
$$

Observing the inclusion

$$
\left\{U_{n}(k) \geq U_{n}\left(k_{0}\right)\right\} \subset\left\{A(k)+B(k)+C(k)-\left(A\left(k_{0}\right)+B\left(k_{0}\right)+C\left(k_{0}\right)\right) \geq E\right\}
$$

we now investigate the other terms $A(k)-A\left(k_{0}\right), B(k)-B\left(k_{0}\right), C(k)-C\left(k_{0}\right)$. A straightforward but tedious calculation yields the decomposition

$$
B(k)-B\left(k_{0}\right)=-B_{1}+B_{2}+B_{3},
$$

where

$$
\begin{aligned}
& B_{1}=\frac{1}{n^{4}}\left(k_{0}-1\right)\left[a_{n, k_{0}}-a_{n, k}\right]\left(\boldsymbol{\mu}_{1}-\boldsymbol{\mu}_{2}\right)^{T} \sum_{i=1}^{k_{0}} \mathbf{w}_{i}, \\
& B_{2}=\frac{1}{n^{4}} k_{0}\left(k_{0}-1\right)\left(k-k_{0}\right)\left(\boldsymbol{\mu}_{1}-\boldsymbol{\mu}_{2}\right)^{T} \sum_{j=k_{0}+1}^{n} \mathbf{w}_{j}, \\
& B_{3}=\frac{1}{n^{4}} k_{0}\left(k_{0}-1\right)(n-k-1)\left(\boldsymbol{\mu}_{1}-\boldsymbol{\mu}_{2}\right)^{T} \sum_{j=k_{0}+1}^{k} \mathbf{w}_{j} .
\end{aligned}
$$

Using (5.25) the term $B_{1}$ can be handled as follows

$$
\begin{aligned}
\mathbb{P}\left\{\left|B_{1}\right| \geq c E\right\} & =\mathbb{P}\left\{\left|\left(\boldsymbol{\mu}_{1}-\boldsymbol{\mu}_{2}\right)^{T} \sum_{i=1}^{k_{0}} \mathbf{w}_{i}\right| \geq c k_{0}\left\|\boldsymbol{\mu}_{1}-\boldsymbol{\mu}_{2}\right\|^{2}\right\} \\
& \leq \mathbb{P}\left\{\left\|\sum_{i=1}^{k_{0}} \mathbf{w}_{i}\right\|^{2} \geq\left(c k_{0}\left\|\boldsymbol{\mu}_{1}-\boldsymbol{\mu}_{2}\right\|\right)^{2}\right\} \\
& \leq \sum_{j=1}^{m} \mathbb{P}\left\{\left|\sum_{i=1}^{k_{0}} W_{i j}\right| \geq \frac{c k_{0}\left\|\boldsymbol{\mu}_{1}-\boldsymbol{\mu}_{2}\right\|}{\sqrt{m}}\right\} \\
& =\sum_{j=1}^{m} \mathbb{P}\left\{\left|\frac{1}{k_{0}} \sum_{i=1}^{k_{0}} W_{i j}\right| \geq c \frac{\left\|\boldsymbol{\mu}_{1}-\boldsymbol{\mu}_{2}\right\|}{\sqrt{m}}\right\} .
\end{aligned}
$$

Note that for each $j=1, \cdots, m$, there exists a position $(a, b)$ such that $W_{i j}$ can be written as

$$
W_{i j}=\dot{X}_{i a} \dot{X}_{i b}-\mathbb{E}\left(X_{i a} X_{i b}\right)=X_{i a} X_{i b}-\mathbb{E}\left(X_{i a} X_{i b}\right)-X_{i a} \bar{X}_{b}-X_{i b} \bar{X}_{a}+\bar{X}_{a} \bar{X}_{b}
$$


and thus we obtain

$$
\frac{1}{k_{0}} \sum_{i=1}^{k_{0}} W_{i j}=\frac{1}{k_{0}} \sum_{i=1}^{k_{0}}\left[X_{i a} X_{i b}-\mathbb{E}\left(X_{i a} X_{i b}\right)\right]-\bar{X}_{k_{0} a} \bar{X}_{b}-\bar{X}_{k_{0} b} \bar{X}_{a}+\bar{X}_{a} \bar{X}_{b} .
$$

With these notations the probability in (5.27) can be further bounded using Lemmas 5.2 and 5.3, that is

$$
\begin{aligned}
& \mathbb{P}\left\{\left|B_{1}\right| \geq c E\right\} \leq m\left[\mathbb{P}\left\{\left|\frac{1}{k_{0}} \sum_{i=1}^{k_{0}}\left[X_{i a} X_{i b}-\mathbb{E}\left(X_{i a} X_{i b}\right)\right]\right| \geq c_{1} \frac{\left\|\boldsymbol{\mu}_{1}-\boldsymbol{\mu}_{2}\right\|}{\sqrt{m}}\right\}+2 \mathbb{P}\left\{\left|\bar{X}_{k_{0} a}\right| \geq c_{2} \sqrt{\frac{\left\|\boldsymbol{\mu}_{1}-\boldsymbol{\mu}_{2}\right\|}{\sqrt{m}}}\right\}\right. \\
& \left.+3 \mathbb{P}\left\{\left|\bar{X}_{b}\right| \geq c_{3} \sqrt{\frac{\left\|\boldsymbol{\mu}_{1}-\boldsymbol{\mu}_{2}\right\|}{\sqrt{m}}}\right\}+\mathbb{P}\left\{\left|\bar{X}_{a}\right| \geq c_{4} \sqrt{\frac{\left\|\boldsymbol{\mu}_{1}-\boldsymbol{\mu}_{2}\right\|}{\sqrt{m}}}\right\}\right] \\
& \leq c_{6} m\left[e^{-c_{7} \min \left\{k_{0} \frac{\left\|\mu_{1}-\mu_{2}\right\|^{2}}{m}, k_{0} \frac{\left\|\mu_{1}-\mu_{2}\right\|}{\sqrt{m}}\right\}}+e^{-c_{7} k_{0} \frac{\left\|\mu_{1}-\mu_{2}\right\|}{\sqrt{m}}}+e^{-c_{7} n \frac{\left\|\mu_{1}-\mu_{2}\right\|}{\sqrt{m}}}\right] \\
& \leq c_{6} p^{2}\left[e^{-c_{7} \min \{\tau, \sqrt{n \tau}\}}+e^{-c_{7} \sqrt{n \tau}}+e^{-c_{7} \sqrt{n \tau}}\right] \leq c_{8} p^{2} e^{-c_{7} \min \{\tau, \sqrt{n \tau}\}},
\end{aligned}
$$

where the last inequality follows from the fact that $k_{0} \frac{\left\|\boldsymbol{\mu}_{1}-\boldsymbol{\mu}_{2}\right\|^{2}}{m} \geq C \tau$ and $k_{0} \frac{\left\|\boldsymbol{\mu}_{1}-\boldsymbol{\mu}_{2}\right\|}{\sqrt{m}} \geq C \sqrt{n \tau}$ if the smallest nonzero entry of $\left(\Sigma_{1}-\Sigma_{2}\right)$ satisfies $\left|\sigma_{1}(a, b)-\sigma_{2}(a, b)\right| \geq \lambda \geq C \sqrt{\frac{\tau}{n}} \frac{n}{k_{0}}$. Note that Assumption 3.3 gives $p^{2} n=o\left(e^{c \tau}\right), p^{2} n=o\left(e^{c \sqrt{n \tau}}\right)$, and therefore it follows that

$$
\mathbb{P}\left\{\left|B_{1}\right| \geq c E\right\}=o\left(\frac{1}{n}\right) \text {. }
$$

Similarly, each component of the vector $B_{2}$ can be represented as

$$
\frac{1}{n-k_{0}} \sum_{i=k_{0}+1}^{n} W_{i j}=\frac{1}{n-k_{0}} \sum_{i=k_{0}+1}^{n}\left[X_{i a} X_{i b}-\mathbb{E}\left(X_{i a} X_{i b}\right)\right]-\bar{Y}_{k_{0} a} \bar{X}_{b}-\bar{Y}_{k_{0} b} \bar{X}_{a}+\bar{X}_{a} \bar{X}_{b}
$$

for some $(a, b) \in \mathcal{P}$. We can find that

$$
\begin{aligned}
\mathbb{P}\left\{\left|B_{2}\right| \geq c E\right\} & =\mathbb{P}\left\{\left|\left(\boldsymbol{\mu}_{1}-\boldsymbol{\mu}_{2}\right)^{T} \sum_{i=k_{0}+1}^{n} \mathbf{w}_{i}\right| \geq c\left(n-k_{0}+n-k-1\right)\left\|\boldsymbol{\mu}_{1}-\boldsymbol{\mu}_{2}\right\|^{2}\right\} \\
& \leq \mathbb{P}\left\{\left\|\boldsymbol{\mu}_{1}-\boldsymbol{\mu}_{2}\right\|\left\|\sum_{i=k_{0}+1}^{n} \mathbf{w}_{i}\right\| \geq c\left(n-k_{0}+n-k-1\right)\left\|\boldsymbol{\mu}_{1}-\boldsymbol{\mu}_{2}\right\|^{2}\right\} \\
& \leq m \cdot \mathbb{P}\left\{\left|\sum_{i=k_{0}+1}^{n} W_{i j}\right| \geq \frac{c\left(n-k_{0}+n-k-1\right)\left\|\boldsymbol{\mu}_{1}-\boldsymbol{\mu}_{2}\right\|}{\sqrt{m}}\right\} \\
& \leq m \cdot \mathbb{P}\left\{\left|\frac{1}{n-k_{0}} \sum_{i=k_{0}+1}^{n} W_{i j}\right| \geq \frac{c\left\|\boldsymbol{\mu}_{1}-\boldsymbol{\mu}_{2}\right\|}{\sqrt{m}}\right\} \\
& \leq c_{1} m\left[e^{-c_{2} \min \left\{\left(n-k_{0}\right) \frac{\left\|\boldsymbol{\mu}_{1}-\boldsymbol{\mu}_{2}\right\|^{2}}{m},\left(n-k_{0}\right) \frac{\left\|\boldsymbol{\mu}_{1}-\boldsymbol{\mu}_{2}\right\|}{\sqrt{m}}\right\}}+e^{-c_{2}\left(n-k_{0}\right) \frac{\left\|\boldsymbol{\mu}_{1}-\boldsymbol{\mu}_{2}\right\|}{\sqrt{m}}}+e^{-c_{2} n \frac{\left\|\boldsymbol{\mu}_{1}-\boldsymbol{\mu}_{2}\right\|}{\sqrt{m}}}\right] \\
& \leq c_{1} p^{2}\left[e^{-c_{3} \min \{\tau, \sqrt{n \tau}\}}+e^{-c_{3} \sqrt{n \tau}}+e^{-c_{3} \sqrt{n \tau}}\right] \leq c_{4} p^{2} e^{-c_{3} \min \{\tau, \sqrt{n \tau}\}},
\end{aligned}
$$


where the last inequality follows from the fact that $\left|\sigma_{1}(a, b)-\sigma_{2}(a, b)\right| \geq C \sqrt{\frac{\tau}{n}} \frac{n}{n-k_{0}}$ for all $(a, b) \in \mathcal{P}$, which implies $\left(n-k_{0}\right) \frac{\left\|\boldsymbol{\mu}_{1}-\boldsymbol{\mu}_{2}\right\|^{2}}{m} \geq C \tau$ and $\left(n-k_{0}\right) \frac{\left\|\boldsymbol{\mu}_{1}-\boldsymbol{\mu}_{2}\right\|}{\sqrt{m}} \geq C \sqrt{n \tau}$. So we get

$$
\mathbb{P}\left\{\left|B_{2}\right| \geq c E\right\}=o\left(\frac{1}{n}\right) .
$$

In order to get a similar result for the term $B_{3}$ we note that

$$
\begin{aligned}
\mathbb{P}\left\{\left|B_{3}\right| \geq c E\right\} & \leq \mathbb{P}\left\{\left\|\boldsymbol{\mu}_{1}-\boldsymbol{\mu}_{2}\right\|^{2} \sum_{j=1}^{m}\left(\sum_{i=k_{0}+1}^{k} W_{i j}\right)^{2} \geq c\left[2\left(k-k_{0}\right)+\frac{\left(k-k_{0}\right)^{2}}{n-k-1}\right]^{2}\left\|\boldsymbol{\mu}_{1}-\boldsymbol{\mu}_{2}\right\|^{4}\right\} \\
& \leq m \mathbb{P}\left\{\left|\sum_{i=k_{0}+1}^{k} W_{i j}\right| \geq c\left[2\left(k-k_{0}\right)+\frac{\left(k-k_{0}\right)^{2}}{n-k-1}\right]\left\|\boldsymbol{\mu}_{1}-\boldsymbol{\mu}_{2}\right\| / \sqrt{m}\right\} \\
& \leq m \cdot \mathbb{P}\left\{\left|\frac{1}{k-k_{0}} \sum_{i=k_{0}+1}^{k} W_{i j}\right| \geq \frac{2 c\left\|\boldsymbol{\mu}_{1}-\boldsymbol{\mu}_{2}\right\|}{\sqrt{m}}\right\} \\
& \leq c_{1} m\left[e^{-c_{2} \min \left\{\left(k-k_{0}\right) \frac{\left\|\boldsymbol{\mu}_{1}-\boldsymbol{\mu}_{2}\right\|^{2}}{m},\left(k-k_{0}\right) \frac{\left\|\boldsymbol{\mu}_{1}-\boldsymbol{\mu}_{2}\right\|}{\sqrt{m}}\right\}}+e^{-c_{2}\left(k-k_{0}\right) \frac{\left\|\boldsymbol{\mu}_{1}-\boldsymbol{\mu}_{2}\right\|}{\sqrt{m}}}+e^{-c_{2} n \frac{\left\|\boldsymbol{\mu}_{1}-\boldsymbol{\mu}_{2}\right\|}{\sqrt{m}}}\right] \\
& \leq c_{1} m\left[e^{-c_{2} \epsilon \min \left\{k_{0} \frac{\left\|\boldsymbol{\mu}_{1}-\boldsymbol{\mu}_{2}\right\|^{2}}{m}, k_{0} \frac{\left\|\boldsymbol{\mu}_{1}-\boldsymbol{\mu}_{2}\right\|}{\sqrt{m}}\right\}}+e^{-c_{2} \epsilon k_{0}} \frac{\left\|\boldsymbol{\mu}_{1}-\boldsymbol{\mu}_{2}\right\|}{\sqrt{m}}+e^{-c_{2} n \frac{\left\|\boldsymbol{\mu}_{1}-\boldsymbol{\mu}_{2}\right\|}{\sqrt{m}}}\right] \\
& \leq c_{3} p^{2} e^{-c_{4} \min \{\tau, \sqrt{n \tau}\}} .
\end{aligned}
$$

By condition (3.1) in Assumption 3.2 and Assumption 3.3, we get

$$
\mathbb{P}\left\{\left|B_{3}\right| \geq c E\right\}=o\left(\frac{1}{n}\right) .
$$

Combining (5.30) and (5.31), we can conclude that

$$
\mathbb{P}\left\{\left|B(k)-B\left(k_{0}\right)\right| \geq c E\right\} \leq c_{1} p^{2} e^{-c_{2} \min \{\tau, \sqrt{n \tau}\}}=o\left(\frac{1}{n}\right)
$$

uniformly with respect to $k \geq k_{0}(1+\varepsilon)$. Similarly, one can see that the estimate

$$
\mathbb{P}\left\{\left|C(k)-C\left(k_{0}\right)\right| \geq c E\right\} \leq c_{1} p^{2} e^{-c_{2} \min \{\tau, \sqrt{n \tau}\}}=o\left(\frac{1}{n}\right) .
$$

Finally, we investigate the terms $A(k)$ and $A\left(k_{0}\right)$ introducing the decomposition

$$
A(k)=A_{1}(k)-2 A_{2}(k)+A_{3}(k),
$$

where

$$
\begin{aligned}
& A_{1}(k)=\frac{1}{n^{4}}(n-k)(n-k-1) \sum_{(i \neq t)=1}^{k} \sum_{i}^{k} \mathbf{w}_{i}^{T} \mathbf{w}_{t}, \quad A_{3}(k)=\frac{1}{n^{4}} k(k-1) \sum_{(j \neq l)=k+1}^{n} \sum_{i=1}^{n} \mathbf{w}_{j}^{T} \mathbf{w}_{l}, \\
& A_{2}(k)=\frac{1}{n^{4}}(k-1)(n-k-1) \sum_{l=k+1}^{k} \mathbf{w}_{i}^{T} \mathbf{w}_{l} .
\end{aligned}
$$


Then

$$
A(k)-A\left(k_{0}\right)=\left[A_{1}(k)-A_{1}\left(k_{0}\right)\right]-2\left[A_{2}(k)-A_{2}\left(k_{0}\right)\right]+\left[A_{3}(k)-A_{3}\left(k_{0}\right)\right] .
$$

In order to get that $\mathbb{P}\left\{\left|A(k)-A\left(k_{0}\right)\right| \geq c E\right\}=o\left(\frac{1}{n}\right)$, it is sufficient to show that $\mathbb{P}\left\{\mid A_{i}(k)-\right.$ $\left.A_{i}\left(k_{0}\right) \mid \geq c E\right\}=o\left(\frac{1}{n}\right), i=1,2,3$. In the following we only show that

$$
\mathbb{P}\left\{\left|A_{1}(k)-A_{1}\left(k_{0}\right)\right| \geq c E\right\} \leq c_{1} p^{2}\left[e^{-c_{2} \tau}+n e^{-c_{2} \sqrt{\tau}}\right]=o\left(\frac{1}{n}\right)
$$

uniformly with respect to $k \geq(1+\varepsilon) k_{0}$. The other two terms can be treated similarly.

Define

$H_{1}=\frac{a_{n, k_{0}}-a_{n, k}}{n^{4}} \sum_{i \neq t}^{k_{0}} \sum_{i}^{k_{0}} \mathbf{w}_{i}^{T} \mathbf{w}_{t}, \quad H_{2}=\frac{a_{n, k}}{n^{4}} \sum_{i \neq t=\left(k_{0}+1\right)}^{k} \sum_{i}^{k} \mathbf{w}_{i}^{T} \mathbf{w}_{t}, \quad H_{3}=\frac{a_{n, k}}{n^{4}} \sum_{i=1}^{k_{0}} \sum_{t=k_{0}+1}^{k} \mathbf{w}_{i}^{T} \mathbf{w}_{t}$,

and note that

$$
A_{1}(k)-A_{1}\left(k_{0}\right)=-H_{1}+H_{2}+2 H_{3} .
$$

First, we obtain for the term $H_{1}$ using (3.1)

$$
\begin{aligned}
& \mathbb{P}\left\{\left|H_{1}\right| \geq c E\right\}=\mathbb{P}\left\{\left|\sum_{i \neq t}^{k_{0}} \sum_{0}^{k_{0}} \mathbf{w}_{i}^{T} \mathbf{w}_{t}\right| \geq c k_{0}\left(k_{0}-1\right)\left\|\boldsymbol{\mu}_{1}-\boldsymbol{\mu}_{2}\right\|^{2}\right\} \\
= & \mathbb{P}\left\{\left|\sum_{j=1}^{m} \sum_{i \neq t}^{k_{0}} \sum_{i \neq t}^{k_{0}} W_{i j} W_{t j}\right| \geq c k_{0}\left(k_{0}-1\right)\left\|\boldsymbol{\mu}_{1}-\boldsymbol{\mu}_{2}\right\|^{2}\right\} \\
\leq & m \cdot \mathbb{P}\left\{\left|\sum_{i \neq t}^{k_{0}} \sum_{k_{0}} W_{i j} W_{t j}\right| \geq c k_{0}\left(k_{0}-1\right) \frac{\left\|\boldsymbol{\mu}_{1}-\boldsymbol{\mu}_{2}\right\|^{2}}{m}\right\} \\
\leq & m \cdot \mathbb{P}\left\{\left|\frac{1}{k_{0}} \sum_{i=1}^{k_{0}} W_{i j}\right| \geq c_{1} \frac{\left\|\boldsymbol{\mu}_{1}-\boldsymbol{\mu}_{2}\right\|}{\sqrt{m}}\right\}+m \cdot \mathbb{P}\left\{\left|\frac{1}{k_{0}} \sum_{i=1}^{k_{0}} W_{i j}^{2}\right| \geq c\left(k_{0}-1\right) \frac{\left\|\boldsymbol{\mu}_{1}-\boldsymbol{\mu}_{2}\right\|^{2}}{m}\right\} \\
\leq & c_{2} p^{2} e^{-c_{3} \min \{\tau, \sqrt{n \tau}\}}+p^{2} \mathbb{P}\left\{\left|\frac{1}{k_{0}} \sum_{i=1}^{k_{0}} W_{i j}^{2}\right| \geq c\left(k_{0}-1\right) \frac{\left\|\boldsymbol{\mu}_{1}-\boldsymbol{\mu}_{2}\right\|^{2}}{m}\right\}
\end{aligned}
$$

where the last inequality follows by similar arguments as used in the derivation of (5.27) and (5.29). For the second term, we use the decomposition (5.28)

$$
\begin{aligned}
& p^{2} \mathbb{P}\left\{\left|\frac{1}{k_{0}} \sum_{i=1}^{k_{0}} W_{i j}^{2}\right| \geq \frac{c}{2}\left(k_{0}-1\right) \frac{\left\|\boldsymbol{\mu}_{1}-\boldsymbol{\mu}_{2}\right\|^{2}}{m}\right\} \\
\leq & p^{2} k_{0} \cdot \mathbb{P}\left\{\left|W_{i j}\right| \geq \sqrt{\left.\frac{c}{2}\left(k_{0}-1\right) \frac{\left\|\boldsymbol{\mu}_{1}-\boldsymbol{\mu}_{2}\right\|}{\sqrt{m}}\right\}}\right. \\
\leq & p^{2} k_{0} \cdot \mathbb{P}\left\{\left|X_{i a} X_{i b}-\mathbb{E}\left(X_{i a} X_{i b}\right)\right| \geq c_{4} \sqrt{k_{0}} \frac{\left\|\boldsymbol{\mu}_{1}-\boldsymbol{\mu}_{2}\right\|}{\sqrt{m}}\right\} \\
& +2 p^{2} k_{0} \cdot \mathbb{P}\left\{\left|X_{i a} \bar{X}_{b}\right| \geq c_{4} \sqrt{k_{0}} \frac{\left\|\boldsymbol{\mu}_{1}-\boldsymbol{\mu}_{2}\right\|}{\sqrt{m}}\right\}+p^{2} k_{0} \cdot \mathbb{P}\left\{\left|\bar{X}_{a} \bar{X}_{b}\right| \geq c_{4} \sqrt{k_{0}} \frac{\left\|\boldsymbol{\mu}_{1}-\boldsymbol{\mu}_{2}\right\|}{\sqrt{m}}\right\} \\
\leq & c_{5} p^{2} k_{0} e^{-c_{6} \sqrt{k_{0}} \frac{\left\|\boldsymbol{\mu}_{1}-\boldsymbol{\mu}_{2}\right\|}{\sqrt{m}}} \leq c_{5} p^{2} n e^{-c_{7} \sqrt{\tau}}=o\left(\frac{1}{n}\right),
\end{aligned}
$$


where the last line uses Lemma 5.1 for the sub-exponential random variable $X_{i a} X_{i b}$ and the probability of this sub-exponential term is also the leading one among the remaining three terms. Moreover, because $\left|\sigma_{1}(a, b)-\sigma_{2}(a, b)\right| \geq C \sqrt{\frac{\tau}{n}} \sqrt{\frac{n}{k_{0}}}$ for all $(a, b) \in \mathcal{P}$ we have $\sqrt{k_{0}} \frac{\left\|\boldsymbol{\mu}_{1}-\boldsymbol{\mu}_{2}\right\|}{\sqrt{m}} \geq C \sqrt{\tau}$. Then the order $o\left(\frac{1}{n}\right)$ comes from the assumptions (3.3) - (3.5). Combining this estimate with (5.34) gives

$$
\mathbb{P}\left(\left|H_{1}\right| \geq c E\right) \leq c_{2} p^{2} e^{-c_{3} \min \{\tau, \sqrt{n \tau}\}}+c_{5} p^{2} n e^{-c_{7} \sqrt{\tau}}=o\left(\frac{1}{n}\right) .
$$

Next for the term $\mathrm{H}_{2}$, similarly we can calculate

$$
\begin{aligned}
& \mathbb{P}\left\{\left|H_{2}\right| \geq c E\right\} \\
= & \mathbb{P}\left\{\left|\sum_{i \neq t=\left(k_{0}+1\right)}^{k} \sum_{i}^{T} \mathbf{w}_{t}\right| \geq c \frac{k_{0}\left(k_{0}-1\right)}{n-k}\left[\frac{n-k}{n-k-1}\left(k-k_{0}\right)+\left(k-k_{0}\right)+\frac{\left(k-k_{0}\right)^{2}}{n-k-1}\right]\left\|\boldsymbol{\mu}_{1}-\boldsymbol{\mu}_{2}\right\|^{2}\right\} \\
\leq & m \cdot \mathbb{P}\left\{\left|\sum_{i \neq t=\left(k_{0}+1\right)}^{k} \sum_{i j}^{k} W_{t j}\right| \geq c \frac{k_{0}\left(k_{0}-1\right)}{n-k}\left[2\left(k-k_{0}\right)+\frac{\left(k-k_{0}\right)^{2}}{n-k-1}\right] \frac{\left\|\boldsymbol{\mu}_{1}-\boldsymbol{\mu}_{2}\right\|^{2}}{m}\right\} \\
\leq & m \cdot \mathbb{P}\left\{\left|\frac{1}{k-k_{0}} \sum_{i=k_{0}+1}^{k} W_{i j}\right| \geq \sqrt{\left.\frac{c}{2} \frac{k_{0}\left(k_{0}-1\right)}{(n-k)}\left[\frac{2}{\left(k-k_{0}\right)}+\frac{1}{n-k-1}\right] \frac{\left\|\boldsymbol{\mu}_{1}-\boldsymbol{\mu}_{2}\right\|}{\sqrt{m}}\right\}}\right. \\
& +m \cdot \mathbb{P}\left\{\left|\frac{1}{k-k_{0}} \sum_{i=k_{0}+1}^{k} W_{i j}^{2}\right| \geq \frac{c}{2} \frac{k_{0}\left(k_{0}-1\right)}{n-k}\left[2+\frac{\left(k-k_{0}\right)}{n-k-1}\right] \frac{\left\|\boldsymbol{\mu}_{1}-\boldsymbol{\mu}_{2}\right\|^{2}}{m}\right\} .
\end{aligned}
$$

Using similar arguments as in the discussion of the term (5.34), the above probability can be further bounded by

$$
\begin{aligned}
& \mathbb{P}\left\{\left|H_{2}\right| \geq c E\right\} \\
& \leq c_{1} m e^{-c_{2}\left(k-k_{0}\right) \min \left\{\frac{k_{0}^{2}}{\left(n-k_{0}\right)\left(k-k_{0}\right)} \frac{\left\|\boldsymbol{\mu}_{1}-\boldsymbol{\mu}_{2}\right\|^{2}}{m}, \sqrt{\frac{k_{0}^{2}}{\left(n-k_{0}\right)\left(k-k_{0}\right)}} \frac{\left\|\boldsymbol{\mu}_{1}-\boldsymbol{\mu}_{2}\right\|}{\sqrt{m}}\right\}}+c_{2} m n e^{-c_{3} \sqrt{\frac{k_{0}^{2}}{n-k_{0}}} \frac{\left\|\boldsymbol{\mu}_{1}-\boldsymbol{\mu}_{2}\right\|}{\sqrt{m}}} \\
& \leq c_{1} p^{2} e^{-c_{4} \min \{\tau, \sqrt{n \tau}\}}+c_{2} p^{2} n e^{-c_{5} \sqrt{\tau}}=o\left(\frac{1}{n}\right)
\end{aligned}
$$

where the last line is due to the observation that if the smallest nonzero entry of $\left(\Sigma_{1}-\Sigma_{2}\right)$ satisfies $\left|\sigma_{1}(a, b)-\sigma_{2}(a, b)\right| \geq C \sqrt{\frac{\tau}{n}} \frac{n}{k_{0}} \sqrt{\frac{n-k_{0}}{k_{0}}}$ for some large $C$ in (3.1), then

$$
\frac{k_{0}^{2}}{\left(n-k_{0}\right)} \frac{\left\|\boldsymbol{\mu}_{1}-\boldsymbol{\mu}_{2}\right\|^{2}}{m} \geq C \tau \text { and } \sqrt{\frac{k_{0}^{2}\left(k-k_{0}\right)}{n-k_{0}}} \frac{\left\|\boldsymbol{\mu}_{1}-\boldsymbol{\mu}_{2}\right\|}{\sqrt{m}} \geq C \sqrt{n \tau} \text {. }
$$

So together with $p^{2} n=o\left(e^{c \tau}\right), p^{2} n=o\left(e^{c \sqrt{n \tau}}\right)$ and $p^{2} n^{2}=o\left(e^{c \sqrt{\tau}}\right)$ in Assumption 3.3, we can find the probability to be of order $o\left(\frac{1}{n}\right)$. For the term $H_{3}$, according to (3.1) in Assumption 
3.2, we have

$$
\begin{aligned}
& \mathbb{P}\left\{\left|H_{3}\right| \geq c E\right\} \leq m \cdot \mathbb{P}\left\{\left|\sum_{i=1}^{k_{0}} \sum_{t=k_{0}+1}^{k} W_{i j} W_{t j}\right| \geq c \frac{k_{0}\left(k_{0}-1\right)}{n-k}\left[2\left(k-k_{0}\right)\right] \frac{\left\|\boldsymbol{\mu}_{1}-\boldsymbol{\mu}_{2}\right\|^{2}}{m}\right\} \\
& \leq m \cdot \mathbb{P}\left\{\left|\frac{\sum_{i=1}^{k_{0}} W_{i j}}{k_{0}}\right| \geq \sqrt{\frac{\left(k_{0}-1\right)}{n-k_{0}}} \frac{\left\|\boldsymbol{\mu}_{1}-\boldsymbol{\mu}_{2}\right\|}{\sqrt{m}}\right\}+m \cdot \mathbb{P}\left\{\left|\frac{\sum_{t=k_{0}+1}^{k} W_{t j}}{k-k_{0}}\right| \geq 2 c \sqrt{\frac{\left(k_{0}-1\right)}{n-k_{0}}} \frac{\left\|\boldsymbol{\mu}_{1}-\boldsymbol{\mu}_{2}\right\|}{\sqrt{m}}\right\} \\
& \leq c_{1} m\left[e^{-c_{2} k_{0} \min \left\{\frac{k_{0}}{n-k_{0}} \frac{\left\|\mu_{1}-\boldsymbol{\mu}_{2}\right\|^{2}}{m}, \sqrt{\frac{k_{0}}{n-k_{0}}} \frac{\left\|\mu_{1}-\boldsymbol{\mu}_{2}\right\|}{\sqrt{m}}\right\}}+e^{-c_{3}\left(k-k_{0}\right) \min \left\{\frac{k_{0}}{n-k_{0}} \frac{\left\|\boldsymbol{\mu}_{1}-\boldsymbol{\mu}_{2}\right\|^{2}}{m}, \sqrt{\frac{k_{0}}{n-k_{0}}} \frac{\left\|\boldsymbol{\mu}_{1}-\boldsymbol{\mu}_{2}\right\|}{\sqrt{m}}\right\}}\right] \\
& \leq c_{1} m e^{-c_{4} \min \{\tau, \sqrt{n \tau}\}}=o\left(\frac{1}{n}\right)
\end{aligned}
$$

when the smallest nonzero entry of $\left(\Sigma_{1}-\Sigma_{2}\right)$ satisfies $\left|\sigma_{1}(a, b)-\sigma_{2}(a, b)\right| \geq C \sqrt{\frac{\tau}{n}} \frac{n}{k_{0}} \sqrt{\frac{n-k_{0}}{k_{0}}}$ for some large $C$ and $p^{2} n=o\left(e^{c \tau}\right), p^{2} n=o\left(e^{c \sqrt{n \tau}}\right)$. Combining these arguments gives

$$
\mathbb{P}\left(\left|A_{\ell}(k)-A_{\ell}\left(k_{0}\right)\right| \geq c E\right) \leq c_{1} p^{2}\left[e^{-c_{2} \tau}+n e^{-c_{2} \sqrt{\tau}}\right]=o\left(\frac{1}{n}\right), \quad \ell=1,2,3,
$$

where we note once again that the cases $\ell=2,3$ follow by similar arguments as given for $\ell=1$. From (5.26), (5.32), (5.33) we therefore obtain (5.24), which proves

$$
\mathbb{P}\left\{\hat{k} \geq(1+\epsilon) k_{0}\right\} \leq c_{5} p^{2} n\left[e^{-c_{6} \tau}+n e^{-c_{6} \sqrt{\tau}}\right] \rightarrow 0 .
$$

By the discussion at the beginning of the proof and (5.23) the assertion of Theorem 3.3 follows.

\subsection{Proof of Corollary 3.1}

The difference in proving Theorem 3.1, 3.2 and 3.3 under Assumption 3.3 and Assumption 3.4 consists only in a different treatment of the terms $C^{(2)}$ in (5.17), $H_{1}$ in (5.34) (and $H_{2}$ in (5.35)), for which we need to make use of the following Proposition 5.1. The proof of this result is postponed to Section 5.5.

Proposition 5.1. Suppose $y_{1}, \cdots, y_{k}\left(k \geq n^{\epsilon}\right.$ for some $\left.0<\epsilon<1\right)$ are independent subexponential random variables. Let $\Delta>\max _{i} \mathbb{E}\left[y_{i}^{2}\right]$. Then for any positive constants $c>0$, $M>0$ there exists a constant $n_{0}=n_{0}(c, \stackrel{i}{M}) \in \mathbb{N}$, such that for all $n \geq n_{0}$.

$$
\mathbb{P}\left\{\frac{1}{k} \sum_{i=1}^{k} y_{i}^{2}>\Delta\right\}<c n^{-M}
$$

First, we discuss the differences in the proof of Theorem 3.1 and look at the term $C^{(2)}$ in (5.17) recalling the representation $\dot{X}_{i a} \dot{X}_{i b}=X_{i a} X_{i b}-X_{i a} \bar{X}_{b}-X_{i b} \bar{X}_{a}+\bar{X}_{a} \bar{X}_{b}$. Proposition 5.1 gives for the sum corresponding to the first term

$$
\mathbb{P}\left\{\frac{1}{k} \sum_{i=1}^{k}\left(X_{i a} X_{i b}\right)^{2}>c \tau\right\} \leq c \cdot n^{-M}, \quad \forall M>0 .
$$


Moreover, for $k=\left\lfloor n^{1 / 2}\right\rfloor+1, \ldots, n-\left\lfloor n^{1 / 2}\right\rfloor-1$ we have

$$
\begin{aligned}
\mathbb{P}\left\{\frac{1}{k} \sum_{i=1}^{k}\left(X_{i a} \bar{X}_{b}\right)^{2}>c \tau\right\} & \leq k \mathbb{P}\left\{\left|X_{i a} \bar{X}_{b}\right|>c \sqrt{\tau}\right\} \\
& \leq n\left[\mathbb{P}\left\{\left|X_{i a} \bar{X}_{b}\right|>c \sqrt{\tau},\left|\bar{X}_{b}\right|>1\right\}+\mathbb{P}\left\{\left|X_{i a} \bar{X}_{b}\right|>c \sqrt{\tau},\left|\bar{X}_{b}\right| \leq 1\right\}\right] \\
& \leq n\left[\mathbb{P}\left\{\left|\bar{X}_{b}\right|>1\right\}+\mathbb{P}\left\{\left|X_{i a}\right|>c \sqrt{\tau}\right\}\right] \leq c_{1} n\left[e^{-c_{2} n}+e^{-c_{3} \tau}\right] \\
\mathbb{P}\left\{\frac{1}{k} \sum_{i=1}^{k}\left(\bar{X}_{a} \bar{X}_{b}\right)^{2}>c \tau\right\} & \leq k \mathbb{P}\left\{\left|\bar{X}_{a} \bar{X}_{b}\right|>c \sqrt{\tau}\right\} \\
& \leq n\left[\mathbb{P}\left\{\left|\bar{X}_{a}\right|>c_{1} \tau^{1 / 4}\right\}+\mathbb{P}\left\{\left|\bar{X}_{b}\right|>c_{2} \tau^{1 / 4}\right\}\right] \leq c_{3} p^{2} n^{2} e^{-c_{4} n \sqrt{\tau}},
\end{aligned}
$$

and the probability in $(5.17)$ can be bounded by

$$
\mathbb{P}\left\{C^{(2)}>c \tau\right\} \leq c_{1}\left[n^{-M}+n^{2} e^{-c_{2} n}+n^{2} e^{-c_{3} \tau}+n^{2} e^{-c_{4} n \sqrt{\tau}}\right]
$$

Consequently, if

$$
p n^{-M} \rightarrow 0, \quad p^{2} n^{2} e^{-c n} \rightarrow 0, \quad p^{2} n^{2} e^{-c \tau} \rightarrow 0
$$

for some small positive constant $c$, it follows that $p^{2} \cdot \mathbb{P}\left\{C^{(2)}>c \tau\right\} \rightarrow 0$. Here $M>0$ could be any large positive constant. These estimates show that (5.7) holds for the case $i=2$ as long as

$$
p n^{-M} \rightarrow 0, \quad p^{2} n^{2} e^{-c n} \rightarrow 0, \quad p^{2} n^{2} e^{-c \tau} \rightarrow 0, \quad p^{2} n e^{-c n^{\frac{1}{4}} \sqrt{\tau}} \rightarrow 0 .
$$

for some small positive constant $c$. Note that these conditions contain (5.15) and that $p n^{-M} \rightarrow 0$ implies $p^{2} n^{2} e^{-c n} \rightarrow 0$ and $p^{2} n e^{-c n^{\frac{1}{4}} \sqrt{\tau}} \rightarrow 0$. Consequently, (3.7) holds if

$$
p n^{-M} \rightarrow 0, \quad p^{2} n^{2} e^{-c \tau} \rightarrow 0,
$$

where $c$ is some small positive constant and $M>0$ could be any large constant.

Next, we discuss the differences in the proof of Theorem 3.2 and look exemplarily at the term $H_{1}$. For the second term in (5.34), recall that

$$
W_{i j}=X_{i a} X_{i b}-\mathbb{E}\left(X_{i a} X_{i b}\right)-X_{i a} \bar{X}_{b}-X_{i b} \bar{X}_{a}+\bar{X}_{a} \bar{X}_{b}
$$

in equation (5.28). Proposition 5.1 gives

$$
\mathbb{P}\left\{\left|\frac{1}{k_{0}} \sum_{i=1}^{k_{0}}\left(X_{i a} X_{i b}-\mathbb{E}\left(X_{i a} X_{i b}\right)\right)^{2}\right| \geq \frac{c}{2}\left(k_{0}-1\right) \frac{\left\|\boldsymbol{\mu}_{1}-\boldsymbol{\mu}_{2}\right\|^{2}}{m}\right\} \leq c n^{-M} .
$$

In addition,

$$
\begin{aligned}
& \mathbb{P}\left\{\left|\frac{1}{k_{0}} \sum_{i=1}^{k_{0}}\left(X_{i a} \bar{X}_{b}\right)^{2}\right| \geq \frac{c}{2}\left(k_{0}-1\right) \frac{\left\|\boldsymbol{\mu}_{1}-\boldsymbol{\mu}_{2}\right\|^{2}}{m}\right\} \leq k_{0} \cdot \mathbb{P}\left\{\left|X_{i a} \bar{X}_{b}\right| \geq c_{4} \sqrt{k_{0}} \frac{\left\|\boldsymbol{\mu}_{1}-\boldsymbol{\mu}_{2}\right\|}{\sqrt{m}}\right\} \\
\leq & n\left[\mathbb{P}\left\{\left|X_{i a} \bar{X}_{b}\right|>c_{4} \sqrt{k_{0}} \frac{\left\|\boldsymbol{\mu}_{1}-\boldsymbol{\mu}_{2}\right\|}{\sqrt{m}},\left|\bar{X}_{b}\right|>1\right\}+\mathbb{P}\left\{\left|X_{i a} \bar{X}_{b}\right|>c_{4} \sqrt{k_{0}} \frac{\left\|\boldsymbol{\mu}_{1}-\boldsymbol{\mu}_{2}\right\|}{\sqrt{m}},\left|\bar{X}_{b}\right| \leq 1\right\}\right] \\
\leq & n\left[\mathbb{P}\left\{\left|\bar{X}_{b}\right|>1\right\}+\mathbb{P}\left\{\left|X_{i a}\right|>c_{4} \sqrt{k_{0}} \frac{\left\|\boldsymbol{\mu}_{1}-\boldsymbol{\mu}_{2}\right\|}{\sqrt{m}}\right\}\right] \leq c_{1} n\left[e^{-c_{2} n}+e^{-c_{3} k_{0} \frac{\left\|\boldsymbol{\mu}_{1}-\boldsymbol{\mu}_{2}\right\|^{2}}{m}}\right],
\end{aligned}
$$


and

$$
\begin{aligned}
\mathbb{P}\left\{\left|\frac{1}{k_{0}} \sum_{i=1}^{k_{0}}\left(\bar{X}_{a} \bar{X}_{b}\right)^{2}\right| \geq \frac{c}{2}\left(k_{0}-1\right) \frac{\left\|\boldsymbol{\mu}_{1}-\boldsymbol{\mu}_{2}\right\|^{2}}{m}\right\} & \leq p^{2} k_{0} \cdot \mathbb{P}\left\{\left|\bar{X}_{a} \bar{X}_{b}\right| \geq c_{4} \sqrt{k_{0}} \frac{\left\|\boldsymbol{\mu}_{1}-\boldsymbol{\mu}_{2}\right\|}{\sqrt{m}}\right\} \\
& \leq c_{1} p^{2} n e^{-c_{2} n \sqrt{k_{0}} \frac{\left\|\boldsymbol{\mu}_{1}-\boldsymbol{\mu}_{2}\right\|}{\sqrt{m}}} .
\end{aligned}
$$

So, when the smallest nonzero entry of $\left(\Sigma_{1}-\Sigma_{2}\right)$ satisfies $\left|\sigma_{1}(a, b)-\sigma_{2}(a, b)\right| \geq C \sqrt{\frac{\tau}{n}} \sqrt{\frac{n}{k_{0}}}$ for some large $C, \sqrt{k_{0}} \frac{\left\|\boldsymbol{\mu}_{1}-\boldsymbol{\mu}_{2}\right\|}{\sqrt{m}} \geq C \sqrt{\tau}$, the second term can be bounded by

$$
\begin{aligned}
\mathbb{P}\left\{\left|\frac{1}{k_{0}} \sum_{i=1}^{k_{0}} W_{i j}^{2}\right| \geq \frac{c\left(k_{0}-1\right)}{2} \frac{\left\|\boldsymbol{\mu}_{1}-\boldsymbol{\mu}_{2}\right\|^{2}}{m}\right\} & \leq c\left[n^{-M}+n e^{-c n}+n e^{-c k_{0} \frac{\left\|\mu_{1}-\mu_{2}\right\|^{2}}{m}}+n e^{-c n \sqrt{k_{0}} \frac{\left\|\boldsymbol{\mu}_{1}-\boldsymbol{\mu}_{2}\right\|}{\sqrt{m}}}\right] \\
& \leq c\left[n^{-M}+n e^{-c n}+n e^{-c \tau}+n e^{-c n \sqrt{\tau}}\right],
\end{aligned}
$$

and we obtain

$$
\mathbb{P}\left\{\left|H_{1}\right| \geq c E\right\} \leq c\left[p^{2} e^{-c \min \{\tau, \sqrt{n \tau}\}}+p^{2} n^{-M}+p^{2} n e^{-c n}+p^{2} n e^{-c \tau}+p^{2} n e^{-c n \sqrt{\tau}}\right]=o\left(\frac{1}{n}\right),
$$

where the last estimate follows from $p n^{-M} \rightarrow 0$ and $p n e^{-c \tau} \rightarrow 0$ in Assumption 3.4.

Finally, the term $H_{2}$ in (5.35) can be treated using the same derivation as in (5.36) and we obtain

$$
\mathbb{P}\left\{\left|H_{2}\right| \geq c E\right\} \leq c_{1} p^{2} e^{-c_{4} \min \{\tau, \sqrt{n \tau}\}}+c_{2} p^{2}\left[n^{-M}+n e^{-c n}+n e^{-c \tau}+n e^{-c n \sqrt{\tau}}\right]=o\left(\frac{1}{n}\right),
$$

where we use the fact that

$$
\frac{k_{0}^{2}}{\left(n-k_{0}\right)} \frac{\left\|\boldsymbol{\mu}_{1}-\boldsymbol{\mu}_{2}\right\|^{2}}{m} \geq C \tau
$$

if the smallest nonzero entry of $\left(\Sigma_{1}-\Sigma_{2}\right)$ satisfies $\left|\sigma_{1}(a, b)-\sigma_{2}(a, b)\right| \geq C \sqrt{\frac{\tau}{n}} \frac{n}{k_{0}} \sqrt{\frac{n-k_{0}}{k_{0}}}$ for some large $C$ in (3.1). Together with the conditions $p n^{-M} \rightarrow 0$ and $p n e^{-c \tau} \rightarrow 0$ from Assumption 3.4 it follows that the probability is of order $o\left(\frac{1}{n}\right)$.

Adjusting the above three terms in the proof of Theorem 3.1, Theorem 3.2 and Theorem 3.3, we complete the proof of Corollary 3.1.

\subsection{Proof of Proposition 5.1}

Denote $C \triangleq \Delta-\frac{1}{k} \sum_{i=1}^{k} \mathbb{E} y_{i}^{2}, C>0$. By Theorem 4.1 in Johnson et al. (1985) and the following remark, we have for any $h>2$

$$
\begin{aligned}
\mathbb{P}\left\{\frac{1}{k} \sum_{i=1}^{k} y_{i}^{2}>\Delta\right\} & =\mathbb{P}\left\{\frac{1}{k} \sum_{i=1}^{k}\left(y_{i}^{2}-\mathbb{E} y_{i}^{2}\right)>C\right\} \leq \frac{1}{C^{h} k^{h}} \mathbb{E}\left[\sum_{i=1}^{k}\left(y_{i}^{2}-\mathbb{E} y_{i}^{2}\right)\right]^{h} \\
& \leq \frac{C_{1}^{h}}{C^{h} k^{h}}\left(\frac{h}{\log h}\right)^{h} \cdot \max \left\{\left[\mathbb{E}\left|\sum_{i=1}^{k}\left(y_{i}^{2}-\mathbb{E} y_{i}^{2}\right)\right|^{2}\right]^{\frac{h}{2}}, \sum_{i=1}^{k} \mathbb{E}\left|y_{i}^{2}-\mathbb{E} y_{i}^{2}\right|^{h}\right\},
\end{aligned}
$$


where $C_{1}$ is an absolute constant. We calculate the upper bounds for the two terms using the fact that the random variables $y_{i}$ are sub-exponential. For $i=1, \ldots, k$ we have

$$
\begin{aligned}
\mathbb{E}\left|y_{i}^{2}-\mathbb{E} y_{i}^{2}\right|^{h} & \leq 2^{h+1} \mathbb{E} y_{i}^{2 h}=2^{h+1}\left[\mathbb{E} y_{i}^{2 h} I\left\{\left|y_{i}\right| \leq h(\log h)^{2}\right\}+\mathbb{E} y_{i}^{2 h} I\left\{\left|y_{i}\right|>h(\log h)^{2}\right\}\right] \\
& \leq 2^{h+1}\left[h^{2 h}(\log h)^{4 h}+c_{1}\right] \leq c_{2} \cdot 2^{h} h^{2 h}(\log h)^{4 h},
\end{aligned}
$$

and thus for any positive integer $M>0$, we can find $h>2$ such that

$$
\frac{C_{1}^{h}}{C^{h} k^{h}}\left(\frac{h}{\log h}\right)^{h} \cdot \sum_{i=1}^{k} \mathbb{E}\left|y_{i}^{2}-\mathbb{E} y_{i}^{2}\right|^{h} \leq c_{3}^{h} k \cdot\left[\frac{(h \log h)^{3}}{k}\right]^{h} \leq c n^{-M},
$$

where the last inequality is due to the fact that $k>n^{\epsilon}$. Moreover, since the random variables $y_{i}$ are independent, we obtain

$$
\left[\mathbb{E}\left|\sum_{i=1}^{k}\left(y_{i}^{2}-\mathbb{E} y_{i}^{2}\right)\right|^{2}\right]^{\frac{h}{2}}=\left[\sum_{i=1}^{k} \mathbb{E}\left(y_{i}^{2}-\mathbb{E} y_{i}^{2}\right)^{2}\right]^{\frac{h}{2}} \leq c_{4}^{h} k^{\frac{h}{2}} .
$$

Therefore, for any positive integer $M>0$, there exists a constant $h$, such that

$$
\frac{C_{1}^{h}}{C^{h} k^{h}}\left(\frac{h}{\log h}\right)^{h} \cdot\left[\mathbb{E}\left|\sum_{i=1}^{k}\left(y_{i}^{2}-\mathbb{E} y_{i}^{2}\right)\right|^{2}\right]^{\frac{h}{2}} \leq c_{5}^{h}\left(\frac{h}{\sqrt{k} \log h}\right)^{h} \leq c n^{-M}
$$

where the last inequality is also based on the fact that $k>n^{\epsilon}$. Combining (5.36), (5.37) and (5.38) the assertion of Proposition 5.1 follows. 